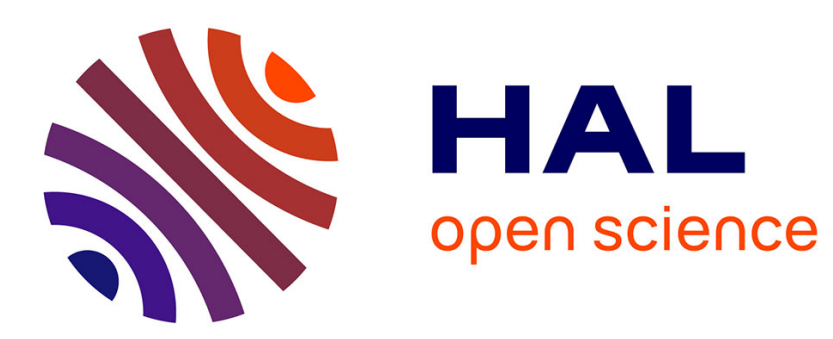

\title{
Symbolic dynamics of two coupled Lorenz maps: from uncoupled regime to synchronisation
}

\author{
Ricardo Coutinho, Bastien Fernandez, Pierre Guiraud
}

\section{To cite this version:}

Ricardo Coutinho, Bastien Fernandez, Pierre Guiraud. Symbolic dynamics of two coupled Lorenz maps: from uncoupled regime to synchronisation. Physica D: Nonlinear Phenomena, 2008, 237 (19), pp.2444-2462. hal-00160086

\section{HAL Id: hal-00160086 https://hal.science/hal-00160086}

Submitted on 4 Jul 2007

HAL is a multi-disciplinary open access archive for the deposit and dissemination of scientific research documents, whether they are published or not. The documents may come from teaching and research institutions in France or abroad, or from public or private research centers.
L'archive ouverte pluridisciplinaire HAL, est destinée au dépôt et à la diffusion de documents scientifiques de niveau recherche, publiés ou non, émanant des établissements d'enseignement et de recherche français ou étrangers, des laboratoires publics ou privés. 


\title{
Symbolic dynamics of two coupled Lorenz maps: from uncoupled regime to synchronisation
}

\author{
R. Coutinho, B. Fernandez $z^{\dagger}$ and P. Guiraud ${ }^{\ddagger}$
}

June 29, 2007

\begin{abstract}
The bounded dynamics of a system of two coupled piecewise affine and chaotic Lorenz maps is studied over the coupling range, from the uncoupled regime where the entropy is maximal, to the synchronized regime where the entropy is minimal. By formulating the problem in terms of symbolic dynamics, bounds on the set of orbit codes (or the set itself, depending on parameters) are determined which describe the way the dynamics is gradually affected as the coupling increases. Proofs rely on monotonicity properties of bounded orbit coordinates with respect to some partial ordering on the corresponding codes. The estimates are translated in terms of (bounds on the) entropy, which are monotonously decreasing with coupling and which are compared to the numerically computed entropy. A good agreement is found which indicates that these bounds capture the essential features of the transition from the uncoupled regime to synchronisation.
\end{abstract}

Keywords: Coupled map lattices; Chaotic dynamics; Symbolic dynamics; Topological entropy; Synchronisation

\section{Introduction}

Lattice dynamical systems are models for the time evolution of systems composed of interacting units [B01, C03, ADGW05]. In many situations, they are governed by the competition between individual and collective terms. This is the case for instance in chains of particles with elastic interaction potential [FM96], in reaction-diffusion systems [K91] and population dynamics or biological regulatory networks [CFLM06, J02]. A natural question is then to describe the dynamics and its changes when the relative strength of individual and interaction terms varies.

In order to be specific, we consider the archetype of discrete time lattice dynamical systems with diffusive interaction, namely Coupled Map Lattices (CML) [CF05, K93]. In the simplest case, their iterations are given by

$$
x_{s}^{t+1}=f\left(x_{s}^{t}\right)+\frac{\epsilon}{2}\left(f\left(x_{s-1}^{t}\right)-2 f\left(x_{s}^{t}\right)+f\left(x_{s+1}^{t}\right)\right)
$$

where $t \in \mathbb{N}$ and where the states $\left\{x_{s}^{t}\right\}$ ( $t$ fixed) are real vectors (or real sequences when the lattice is infinite). The map $f$ is a real map and the number $\epsilon \in[0,1]$ denotes the coupling parameter. In view of the arguments above, the CML can be thought of as the action of the individual term $\left\{x_{s}\right\}_{s \in \mathbb{Z}} \mapsto\left\{f\left(x_{s}\right)\right\}_{s \in \mathbb{Z}}$ which represents on-site forcing, followed by the coupling term $\left\{x_{s}\right\}_{s \in \mathbb{Z}} \mapsto\left\{(1-\epsilon) x_{s}+\frac{\epsilon}{2}\left(x_{s-1}+x_{s+1}\right)\right\}_{s \in \mathbb{Z}}$, a convex combination which models a diffusive coupling.

\footnotetext{
* Group of Mathematical Physics of the University of Lisbon and Department of Mathematics, Instituto Superior Técnico (T U Lisbon), Av. Rovisco Pais 1049-001, Lisboa Codex Portugal, Ricardo.Coutinho@math.ist.utl.pt

${ }^{\dagger}$ Centre de Physique Théorique, CNRS \& Universités de Marseille I et II, et de Toulon, Campus de Luminy Case 907,13288 Marseille CEDEX 09 France, Bastien.Fernandez@cpt.univ-mrs.fr

$\ddagger$ Centro de Modelamiento Matemático, Av. Blanco Encalada 2120 Piso 7, Santiago de Chile

$\S$ Present address: Departamento de Estadística, Fac. Ciencias, Universidad de Valparaíso, Gran Bretaña 1111, Valparaíso, Chile, pierre.guiraud@uv.cl
} 
From a rigorous mathematical point of view, the dynamics of CML has been described in various cases of individual maps and coupling parameter domains, see for instance [CAV00, CF97b] for bistable maps, [D99, FJ04] for unimodal maps and [AF00, JJ02, KL04] for expanding maps. In the case of chaotic (uniformly hyperbolic) individual maps, the dynamics is now fairly understood either when the coupling is sufficiently weak (perturbations of the uncoupled system) [AF00, KL04] or sufficiently strong [JJ02] (synchronisation). However, for intermediate couplings, the dynamics is not so well-known.

The goal of this paper is to develop a description of the dynamics of CML with chaotic individual map, over the whole coupling range. Such CML have been employed to simulate space-time chaos in turbulent systems, see eg. [MYDN01] and the chapter by Kaneko in [K93]. From a theoretical viewpoint, chaotic CML are motivated by the endeavour to prove the occurrence of phase transitions in deterministic lattice dynamical systems [LJ98, MH93]. However, excepted in specially designed CML [GM00, BK06], phase transitions in CML have not been proved up to date. For a detailed discussion on this problem, we refer to [CF05].

Proving that phase transitions exist is related to determining the natural invariant measure(s), the so-called SRB measure(s). Together with an appropriate definition in infinite lattices, the existence of SRB measure has been the central problem of mathematical studies on CML for the last two decades. Indeed, when the phase space is infinite dimensional, the analysis of invariant measures is a delicate task [CF05, KL04]. Moreover, SRB measures have been proved to exist only for weak couplings where perturbation techniques apply.

When the coupling is not so weak, the a priori simpler question of determining the support of this measure (ie. the maximal invariant set) remains also open. In this case, since the coupling operator tends to bring the configurations closer to the diagonal (the set of constant configurations $x_{s}=x$ for all $s$ ), significant changes in the maximal invariant set occur. When the coupling is strong, this set is included in the diagonal and the CML is said to synchronize (see [JJ02] for the synchronisation condition in globally coupled systems). Actually, the occurrence of synchronisation depend on several factors such as the evolution rule, the individual map (regularity), the lattice size and the boundary conditions.

In order to describe the dynamics for all couplings, we shall limit ourselves to the case of a CML of 2 sites ${ }^{1}$ with piecewise affine individual maps. The maps have been chosen so as to allow explicit calculations and to get best estimates. Our analysis however does not rely on this assumption and, in principle, the results extend by continuity to systems with piecewise smooth individual map. On the other hand, the analysis relies on the assumption of a lattice of 2 sites. For larger lattices, a specific analysis needs to be developed. Hopefully, the method and tools introduced here will be of use for the study of larger lattices.

Our analysis of the CML dynamics relies on symbolic dynamics. This means that an admissibility condition is established which characterizes symbolic sequences associated with bounded orbits. (This implies a (natural) coding of orbits and formally solving the recurrence generated by the CML.) The analysis then consists in determining the set of symbolic sequences which satisfy the admissibility condition. In practice, there are actually two admissibility conditions; one applies for weak couplings, where the CML is expanding, and the other applies for strong couplings where the CML has one contracting and one expanding direction.

Except for the boundaries of the coupling range where all admissible sequences can be computed, for arbitrary couplings only estimates are established. More precisely, by considering one-parameter families of sets, a lower bound on the set of admissible sequences is obtained by computing the largest set in the family for which all sequences are admissible. Similarly an upper bound is obtained by computing the smallest set in the family which contain all admissible sequences.

The families are defined so that the parameter controls geometric properties of corresponding orbits ${ }^{2}$. Moreover the parameter values associated with the bounds depend monotonously on the coupling parameter. Therefore these estimates provide a qualitative description of the way the dynamics is gradually affected as the coupling increases from the uncoupled limit to maximal coupling.

In order to quantify these estimates and to appreciate their relevance, subsequent bounds on the entropy are computed and compared with the entropy obtained by numerically computing the number of periodic

\footnotetext{
${ }^{1}$ equivalently, we only consider periodic configurations with period 2 in infinite lattices

2 in particular of their sojourn times in regions of phase space where the orbit point coordinates lie on distinct sides of the individual map discontinuity
} 
points. A good agreement is found which indicates that the bounds capture the essential features of the transition from the uncoupled regime to synchronisation.

In complement to these results, we would like to mention that estimates on the topological entropy in a CML of 2 sites with Tchebyscheff individual maps have been obtained in [DL05] by using periodic orbit theory.

The paper is organized as follows. The dynamical system and the associated symbolic dynamics are firstly introduced (section 2). Then results on numerical computations are presented and analyzed as a motivation for the analysis to follow (section 3). Section 4 contains the analysis of the weak coupling regime where the CML is expanding. A previously obtained lower bound in [FG04] is (largely) improved in two steps and compared to the numerically computed entropy. In the limit $a \rightarrow 2$ (where $a$ is the slope of the individual map), the lower bound becomes trivial. In order to prevent this effect, another family of sets is introduced which implies another lower bound on bounded orbit codes with non trivial behaviour in the previous limit. Section 5 deals with the strong coupling regime where the CML has one contracting direction. A strong restriction on symbolic sequences is firstly shown to apply globally in this domain. Then, as for weak coupling (upper and) lower bound(s) are obtained which show good agreement with the numerical entropy. Finally, the existence of a synchronized regime is established where the only admissible sequences are those corresponding to orbits on the diagonal and to a 2-periodic orbit off diagonal.

\section{The CML and its symbolic dynamics}

\subsection{Two coupled Lorenz maps}

We consider CML of two sites and piecewise affine and expanding individual map. The phase space is the plane $\mathbb{R}^{2}$ and the relation (1) becomes

$$
\left\{\begin{array}{l}
x_{0}^{t+1}=(1-\epsilon) f_{a}\left(x_{0}^{t}\right)+\epsilon f_{a}\left(x_{1}^{t}\right) \\
x_{1}^{t+1}=(1-\epsilon) f_{a}\left(x_{1}^{t}\right)+\epsilon f_{a}\left(x_{0}^{t}\right)
\end{array}\right.
$$

This system of iterations can be viewed as the action $\left(x_{0}^{t+1}, x_{1}^{t+1}\right)=F_{a, \epsilon}\left(x_{0}^{t}, x_{1}^{t}\right)$ of the mapping denoted by $F_{a, \epsilon}$. We shall restrict the coupling parameter $\epsilon$ range to the interval $[0,1 / 2]$ because there is a simple relationship between the orbits of $F_{a, \epsilon}$ and those of $F_{a, 1-\epsilon}$ [D99].

The individual map is a piecewise affine Lorenz map [GH83], namely it is defined by

$$
f_{a}(x)=\left\{\begin{array}{ll}
a x & \text { if } \quad x<1 / 2 \\
a x+1-a & \text { if } \quad x \geqslant 1 / 2
\end{array} \quad x \in \mathbb{R}\right.
$$

In particular, the points 0 and 1 are fixed points and, expected at the discontinuity $x=1 / 2$, the graph of $f_{a}$ is symmetric with respect to the point $(1 / 2,1 / 2)$.

All along the paper, we assume that the slope satisfies $a>2$. As a consequence, the repeller of $f_{a}$, namely the set of points $x \in \mathbb{R}$ with bounded (forward) orbit $\left\{f_{a}^{t}(x)\right\}_{t \in \mathbb{N}}$, is a Cantor set included in $[0,1]$. Moreover, the dynamics in this set can simply be described in terms of symbolic dynamics.

Indeed consider the natural coding which consists in associating the symbol 0 with points smaller than $1 / 2$ and the symbol 1 with points larger than or equal to $1 / 2$. In this way a symbolic sequence $\left\{\vartheta^{t}\right\}_{t \in \mathbb{N}} \in\{0,1\}^{\mathbb{N}}$ can be associated with any (forward) orbit of $f_{a}$. Another consequence of assuming $a>2$ is that the restriction of $f_{a}$ to its repeller is topologically conjugated to the full shift $\left(\{0,1\}^{\mathbb{N}}, \tilde{\sigma}\right)$ where $\tilde{\sigma}$ is the left shift: $(\tilde{\sigma} \vartheta)^{t}=\vartheta^{t+1}, t \in \mathbb{N}$. (The set $\{0,1\}^{\mathbb{N}}$ has been endowed with product topology.) In particular the (topological) entropy of the individual system is $\log 2$.

For the sake of completeness, we mention that when $a<2$, the symbolic dynamics depends on $a$ and has been described using kneading theory [KStP01]. In addition, a complete statistical description of the CML for $a=2$ is presented in [KKN92].

As a preliminary remark to the next section, we mention that since every pre-image of a point in the repeller belongs to this set, the repeller coincides with the set of points $x$ with bounded (forward and backward) orbit $\left\{f_{a}^{t}(x)\right\}_{t \in \mathbb{Z}}$, the so-called maximal hyperbolic set. In particular, points in the maximal hyperbolic set are entirely characterized by their forward code $\left\{\vartheta^{t}\right\}_{t \geqslant 0}$. 


\subsection{Symbolic dynamics of the CML}

Following the individual map coding, a coding of the CML (2) is based on assigning a symbol $\theta_{0} \theta_{1}$ (more precisely a pair of symbols) to points $\left(x_{0}, x_{1}\right)$ in the plane according to the location of their coordinates with respect to $1 / 2$ (see Figure 1 )

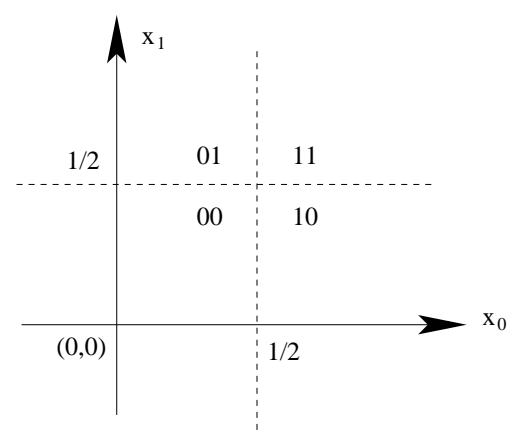

Figure 1: The partition of the CML phase space into 4 atoms labeled by 00, 01, 10 and 11.

- 00 is associated with points $\left(x_{0}, x_{1}\right)$ such that $x_{0}<1 / 2$ and $x_{1}<1 / 2$,

- 01 is associated with points such that $x_{0}<1 / 2$ and $x_{1} \geqslant 1 / 2$,

- etc.

By iterating this process, a (forward) code - denoted by $\theta:=\left\{\theta_{0}^{t} \theta_{1}^{t}\right\}_{t \in \mathbb{N}}-$ can be associated with any orbit $\left\{\left(x_{0}^{t}, x_{1}^{t}\right)\right\}_{t \in \mathbb{N}}$. The set of symbolic sequences $\{01,00,11,10\}^{\mathbb{N}}$ is endowed with the product topology.

For convenience in the sequel, we shall employ the symbol $\theta^{t}$ to denote $\theta_{0}^{t} \theta_{1}^{t}$. We shall also use the following definitions. A word within a symbolic sequence (or the sequence itself) is said to be homogeneous (resp. heterogeneous) if every symbol is either 00 or 11 (resp. either 01 or 10).

As announced in the introduction, the strategy in this paper is to substitute the description of bounded orbits of the CML by the description of symbolic sequences which satisfy the so-called admissibility condition. In order to present this condition we firstly need to study the derivative of $F_{a, \epsilon}$.

The derivative $F_{a, \epsilon}^{\prime}$ is a constant map with two eigenvalues, namely a (the individual map's slope) whose associated eigendirection is the diagonal, and $b:=a(1-2 \epsilon)$ with eigendirection orthogonal to the diagonal. It follows that

- the CML is expanding when $b>1$ (weak coupling domain)

- the CML is hyperbolic (with one expanding and one contracting direction) when $0<b<1$ (strong coupling domain).

For convenience in the sequel, we shall use the parameter $b$ as a coupling parameter, instead of $\epsilon$. Accordingly, the mapping will be denoted by $F_{a, b}$ with $a$ and $b$ considered as independent parameters.

\subsubsection{Weak coupling domain}

In the weak coupling domain $b>1$, the maximal hyperbolic set $K$ of the CML coincides with its repeller (because the CML is onto and expanding). Moreover the CML on its repeller is conjugated ${ }^{3}$ to the shift $\sigma$ on the set of (forward) symbolic sequences $\theta \in\{01,00,11,10\}^{\mathbb{N}}$ which satisfy the admissibility condition

$$
\theta_{s}^{t}=H \circ \Psi_{e}\left(\sigma^{t} R^{s} \theta\right), s \in\{0,1\}, t \geqslant 0
$$

\footnotetext{
${ }^{3}$ We use the term conjugated when the conjugacy from the set of admissible sequences to the repeller is a uniformly continuous bijection and we use the term topologically conjugated when this map is a homeomorphism. Both cases can occur depending whether or not the set of admissible sequences is compact.
} 
(see [FG04] for further details). Here $H$ is the (right continuous) Heaviside function and $R$ is the spatial flip $R \theta=\left\{\theta_{1}^{t} \theta_{0}^{t}\right\}_{t \geqslant 0}$. The symbol $\sigma$ denotes the left shift $\sigma \theta=\left\{\theta_{0}^{t+1} \theta_{1}^{t+1}\right\}_{t \geqslant 0}$ and the function $\Psi_{e}$ is defined by $\Psi_{e}(\theta)=S(\theta)+D_{e}(\theta)$ where

$$
S(\theta)=\sum_{k=0}^{\infty} a^{-k}\left(\theta_{0}^{k}+\theta_{1}^{k}-1\right) \quad \text { and } \quad D_{e}(\theta)=\sum_{k=0}^{\infty} b^{-k}\left(\theta_{0}^{k}-\theta_{1}^{k}\right)
$$

In short terms the condition (3) can be obtained as follows. Firstly one solves the induction associated with the CML for a bounded orbit with a prescribed symbolic sequence. Then the admissibility condition is obtained as the condition which imposes that the code computed by using the solution coincides with the prescribed symbolic sequence.

The admissibility condition possesses the following symmetries. The sequence $\theta$ satisfies the condition (3) iff $R \theta$ satisfies this condition. Moreover, if $\theta$ satisfies (3) and if $\Psi_{e}\left(\sigma^{t} R^{s} \theta\right) \neq 0$ for all $s$ and $t$, then the sequence $1-\theta:=\left\{\left(1-\theta_{0}^{t}\right)\left(1-\theta_{1}^{t}\right)\right\}_{t \in \mathbb{N}}$ satisfies $(3)$. The same properties hold for the strong coupling domain admissibility condition $\left(3^{\prime}\right)$ below.

If a sequence $\theta$ satisfies the condition (3) then the corresponding orbit coordinates write

$$
x_{s}^{t}=\frac{1}{2}+\frac{a-1}{2 a} \Psi_{e}\left(\sigma^{t} R^{s} \theta\right), s \in\{0,1\}, t \geqslant 0
$$

In particular, the previous condition $\Psi_{e}\left(\sigma^{t} R^{s} \theta\right) \neq 0$ simply imposes that no orbit coordinates can lie on the discontinuity $1 / 2$.

An analysis of the condition (3) shows that all sequences in $\{01,00,11,10\}^{\mathbb{N}}$ are admissible iff $b>2$ [FG04]. Accordingly, the analysis in this paper is limited to the domain $0<b<2$.

\subsubsection{Strong coupling domain}

In the weak coupling domain, the bounded dynamics is entirely characterized by forward codes, just as for the individual system. On the opposite, in the strong coupling domain (ie. when $0<b<1$ ), one needs to consider the forward and backward codes $\theta:=\left\{\theta_{0}^{t} \theta_{1}^{t}\right\}_{t \in \mathbb{Z}}$. Similarly as before, in the strong coupling domain, one proves that the maximal hyperbolic set can be described by using symbolic dynamics. Consider the function $\Psi_{h}(\theta)=S(\theta)+D_{h}(\theta)$ where $S$ is as before and where the function $D_{h}$ is defined by (notice the change of index sign in the summation)

$$
D_{h}(\theta)=\sum_{k=1}^{\infty} b^{k}\left(\theta_{1}^{-k}-\theta_{0}^{-k}\right)
$$

Proposition 2.1. Let $a>2$ and $0<b<1$ be arbitrary. A sequence $\left\{\left(x_{0}^{t}, x_{1}^{t}\right)\right\}_{t \in \mathbb{Z}}$ is a bounded orbit of the $C M L$ iff we have $x_{s}^{t}=\frac{1}{2}+\frac{a-1}{2 a} \Psi_{h}\left(\sigma^{t} R^{s} \theta\right)(s \in\{0,1\}, t \in \mathbb{Z})$ where $\theta \in\{01,00,11,10\}^{\mathbb{Z}}$ satisfies the condition

$$
\theta_{s}^{t}=H \circ \Psi_{h}\left(\sigma^{t} R^{s} \theta\right), s \in\{0,1\}, t \in \mathbb{Z}
$$

Proof: Consider the Fourier variables $s^{t}=\frac{x_{0}^{t}+x_{1}^{t}}{2}$ and $d^{t}=\frac{x_{0}^{t}-x_{1}^{t}}{2}$. From the definition of $F_{a, b}$ we have

$$
s^{t+1}=a s^{t}+(1-a) \frac{\theta_{0}^{t}+\theta_{1}^{t}}{2} \quad \text { and } \quad d^{t+1}=b d^{t}+\frac{1-a}{a} b \frac{\theta_{0}^{t}-\theta_{1}^{t}}{2} .
$$

Assuming that $\left\{\left(x_{0}^{t}, x_{1}^{t}\right)\right\}_{t \in \mathbb{Z}}$ is a bounded orbit, one can solve these inductions to obtain the following relations

$$
s^{t}=\frac{1}{2}+\frac{a-1}{2 a} S\left(\sigma^{t} \theta\right) \quad \text { and } \quad d^{t}=\frac{a-1}{2 a} D_{h}\left(\sigma^{t} \theta\right)
$$

for all $t \in \mathbb{Z}$. The expression of $x_{s}^{t}$ then follows from the inversion formulas $\left\{\begin{array}{l}x_{0}^{t}=s^{t}+d^{t} \\ x_{1}^{t}=s^{t}-d^{t}\end{array}\right.$. The rest of the proof is similar to the proof of Proposition 2.1 in [FG04].

Prior to the mathematical analysis to follow, we present in the next section results on the entropy of the CML computed by numerically determining all admissible symbolic sequences (either all forward sequences satisfying the condition (3) when the coupling is weak or all forward and backward sequences satisfying $\left(3^{\prime}\right)$ when the coupling is strong). 


\section{Numerical computation of the entropy}

\subsection{Computing the entropy}

In some sense the complexity of a dynamical system can be quantified by a real number, its the (topological) entropy. In practice, the entropy of the dynamical system $(X, \mathcal{T})$ where $X$ is a topological space and $\mathcal{T}: X \rightarrow X$ is a mapping, can be computed by using Bowen's formula, see eg. [R99]

$$
h(X, \mathcal{T})=\limsup _{n \rightarrow \infty} \frac{\log P_{n}}{n}
$$

where $P_{n}$ is the number of periodic points with (least) period $n$. In our case, computing $P_{n}$ amounts to testing the admissibility of $n$-periodic symbolic sequences.

Strictly speaking, Bowen's formula has been proved in a limited number of cases which do not include the present CML. Nevertheless in all our numerical computations the quantity $\frac{\log P_{n}}{n}$ converges when $n$ increases (see below). Moreover, it lies within the range of analytic estimates when $n$ is large. Therefore, we believe that $\limsup _{n \rightarrow \infty} \frac{\log P_{n}}{n}$ is a good indicator of the CML entropy.

In order to compute this entropy, we have developed an algorithm which tests the admissibility of every $n$-periodic symbolic sequence. Actually, by using symmetries ${ }^{4}$ the algorithm has been optimized so as to test only once the admissibility in the orbits $\left\{\sigma^{t} R^{s} \theta\right\}$ and $\left\{1-\sigma^{t} R^{s} \theta\right\}$. Thanks to the CML being piecewise affine, the admissibility condition (orbit point expression) is explicit and this largely reduces computation times. Yet, computing the 2000 points $\frac{\log P_{15}}{15}$ composing the curves presented below requires approximatively 12 hours by a cluster of 10 processors each with characteristics $1.6 \mathrm{GHz} / 64 \mathrm{Mb}$.

In all experiments, the quantity $\frac{\log P_{n}}{n}$ converges rapidly when $n$ increases. Precisely, independently of $a$ and $b$, the quantity $\left|\frac{\log P_{n}}{n}-\frac{\log P_{n-1}}{n-1}\right|$ decreases with $n$ and the relative difference is smaller than $5 \%$ for $n=15$. As shown on Figure 2, this difference tends to be larger for larger couplings, and it is negligible for the considered values of $n$ when the coupling is small.

\subsection{Numerical results}

The graphs of $\frac{\log P_{15}}{15}$ versus coupling parameter for several values of $a$ is reported on Figure 3 . The picture shows that, independently of $a$, the entropy decreases when the coupling strength increases, excepted at the boundaries where it remains constant. The left plateau corresponds to the entropy of the uncoupled system $(\log 4 \simeq 1.4)$ and the right plateau corresponds to the entropy of synchronized (individual) system $(\log 2 \simeq 0.7)$. In between, the entropy is monotonous and smooth, including across the transition point $b=1$ from expanding to hyperbolic, and possibly expected in the neighbourhood of $b=1 / 2$ where irregularities may appear depending upon numerical accuracy (see next paragraph below). In addition, the figure indicates that the entropy should increase with the individual slope $a$ (and $b$ fixed). It also suggests that the entropy approaches a plateau at $\log 3 \simeq 1.1$ in the left neighbourhood of $b=1$ when $a$ diverges. This property will be confirmed by the mathematical analysis of Section 5 .

In the neighbourhood of $b=1 / 2$ the variations are more subtle. Depending on $n$ and on $a$, the quantity $\frac{\log P_{n}}{n}$ may be locally increasing with coupling strength, see Figure 4 . Local increases seem to be due to finite size effects. We have checked that they are certainly not due to roundoff errors. In all experiments they have disappeared for larger $n$ and this suggests that the entropy is globally decreasing. This suggestion however is to be moderated by the existence in the literature of examples of lattice dynamical systems (also with 2 sites) where the complexity has been shown to be larger for larger coupling parameter [CKNS04]. The problem of entropy global decrease in lattice dynamical systems therefore requires further attention.

Summarizing, the CML complexity smoothly and monotonously varies when the coupling strength increases from uncoupled to synchronized regimes. Some of these features have been mathematically proved in [FG04].

\footnotetext{
${ }^{4}$ ie. the fact that if $\theta$ satisfies the admissibility condition and no orbit point lies on discontinuity lines, then $1-\theta$ where $1-00=11,1-10=01$, etc is also admissible. When computing $P_{n}$, we do not count orbits with points on discontinuity lines and this seems not to affect the entropy.
} 


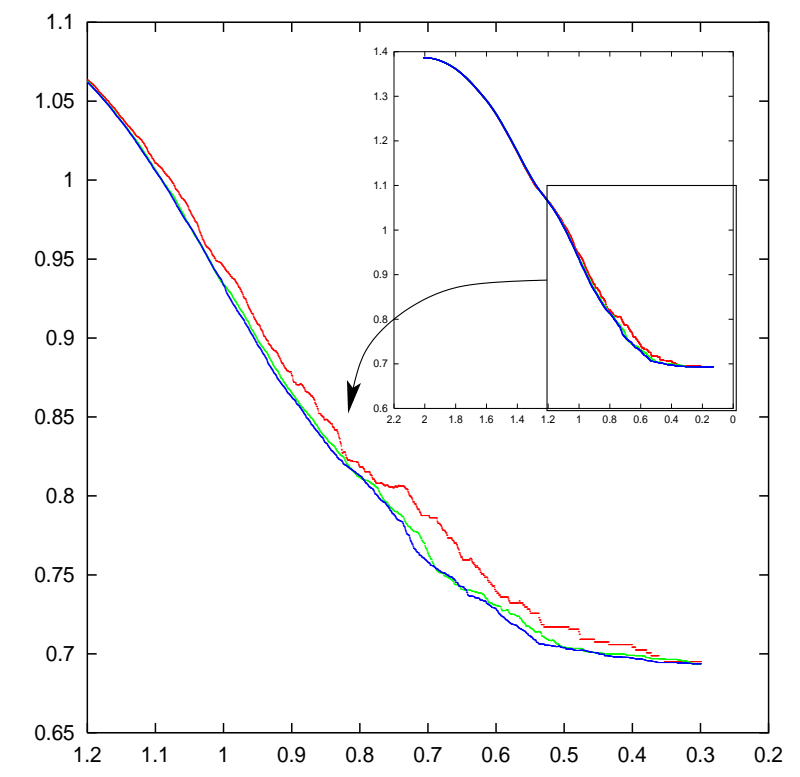

Figure 2: The quantity $\frac{\log P_{n}}{n}$ as a function of $b$ for $a=2.5$ and 3 values of $n: n=11$ (upper/red curve), $n=14$ (intermediate/green curve) and $n=15$ (lower/blue curve). The horizontal axis have been reversed so as to comply with the orientation of the original coupling parameter $\epsilon$. Main picture: $b$ ranges from 0.3 to 1.2 . Inset: $b$ ranges from 0.2 to 2 .

- When $b>2$, the CML is topologically conjugated to the uncoupled system $(b=a)$. In other words all symbolic sequences are admissible and we have a plateau at $\log 4$ for the entropy.

- When $b<2$, some sequences are pruned (in the sense of [CAMTV05]) and the entropy is an increasing function of $b$ in the (left) neighbourhood of $b=2$.

The proof of entropy monotonicity relies in proving monotonicity of the admissibility of symbolic sequences. In principle, it could be improved in order to prove monotonicity on a larger neighbourhood of $b=2$. However, Figure 4 suggests that it cannot apply to all $b$ because the admissibility of some periodic symbolic sequences may not be monotonous when $b$ is close to $1 / 2$. Consequently, the simplest way to prove monotonicity for all couplings would be to show that the set of codes becoming admissible when $b$ decreases has smaller cardinality than the set of codes simultaneously becoming non-admissible. This remains an open problem.

\section{Lower bounds in the weak coupling domain}

A proof of entropy monotonicity for all couplings being out of reach, we adopted another strategy in [FG04]. We showed that the entropy lies between two decreasing step functions of $\epsilon$ with countably many steps. When $b$ is close to 2 these bounds are sufficiently accurate to reflect the numerically computed entropy behaviour. In particular, they converge (with the same slope) to $\log 4$ when $b$ tends to 2 . However when $b$ is close to 1 , the gap between the upper and the lower bound becomes relatively large and the lower bound becomes trivial.

In this section, we improve this lower bound, especially when $b$ is close to 1 , and we show that the entropy is bounded below by a right continuous decreasing function ${ }^{5}$. Actually we obtain a first bound (which we improve in some cases). This bound turns out to provide a good approximation of the entropy when $a$ is large. However, it becomes poor when $a$ is close to 2 . In order to counteract this effect, we construct another lower bound which improves the first bound in this domain.

\footnotetext{
${ }^{5}$ a left continuous increasing function when expressed in terms of $b$
} 


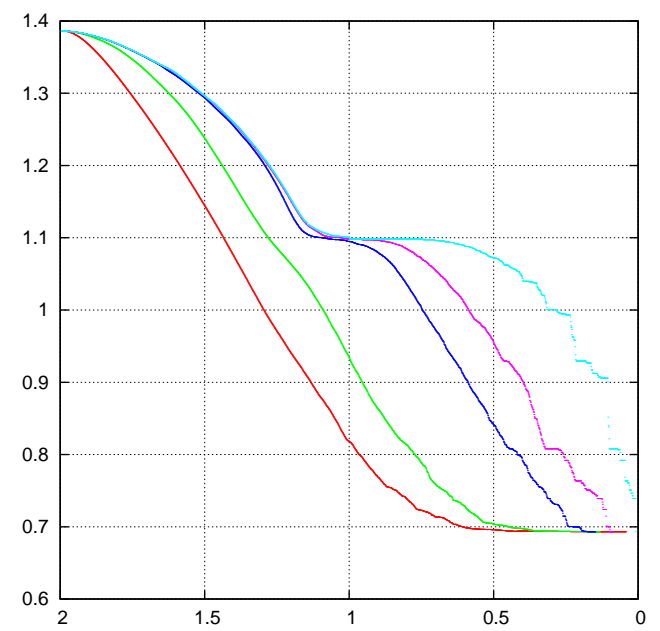

Figure 3: Entropy $\frac{\log P_{15}}{15}$ of the CML as a function of $b$ for 5 values of $a$. From bottom to top $a=2.1$ (lower/red curve), $a=2.5$ (green curve - same as the lower curve in the inset in Figure 2), $a=5$ (dark blue curve), $a=10$ (magenta curve) and $a=100$ (light blue/upper curve).

The proofs rely on comparisons of images under $\Psi_{e}$ of symbolic sequences only differing at two consecutive sites (partial ordering). The strategy is the same for the two bounds. We consider a one-parameter family of sets of symbolic sequences. Given $a$ and $b$, we compute the parameter which corresponds to the largest admissible set in such family (ie. the largest set for which all sequences satisfy (3)). For the first bound and special values of parameters, we use other comparisons on (images under $\Psi_{e}$ of) symbolic sequences to enlarge the admissible set and increase its entropy. iff

We consider the family say $\left\{\Omega_{\nu}\right\}_{\nu \in[0,1]}$ defined as follows. A sequence in $\{01,00,11,10\}^{\mathbb{N}}$ belongs to $\Omega_{\nu}$

$$
\left|\sum_{t=t_{1}}^{t_{2}-1}\left(\theta_{0}^{t}-\theta_{1}^{t}\right)\right| \leqslant\left\lceil\left(t_{2}-t_{1}\right) \nu\right\rceil \quad \forall t_{1}<t_{2} \in \mathbb{N}
$$

This inequality imposes restrictions on the occurrence of identical heterogeneous symbols. For instance, any word of length $k$ containing only the symbols 00,11 and 10 has at most $\lceil k \nu\rceil$ symbols 10 . A word may have more such symbols provided that they are compensated by 01 . From the geometrical point of view it means that the sojourn time in any heterogeneous atom is constrained, possibly depending on visits to the complementary heterogeneous atom.

As a side comment we mention that the lower bound in [FG04] has been obtained by considering only the sets $\Omega_{\frac{n}{n+1}}, n \in \mathbb{N}$. The set $\Omega_{\frac{n}{n+1}}$ consists of sequences for which the length of every word $10^{k}$ and $01^{k}$ does not exceed $n$, independently of any other symbol.

The largest value of $\nu$ such that all sequences in $\Omega_{\nu}$ satisfy the admissibility condition (3) has been computed in Appendix A. Moreover, its dependence on the parameters $a$ and $b$ has been determined. These results are summarized in the next statement.

Theorem 4.1. For every $a>2$ and $1<b<2$, there exists $\nu(a, b) \in[0,1)$ such that for every $\nu<\nu(a, b)$, every sequence in $\Omega_{\nu}$ is the code of some bounded orbit. In addition for every $\nu>\nu(a, b)$ there are sequences in $\Omega_{\nu}$ not corresponding to any bounded orbit code.

The map $b \mapsto \nu(a, b)$ (a fixed) is an increasing Devil's staircase with range $[0,1)$.

The map $a \mapsto \nu(a, b)$ (b fixed) is an increasing Devil's staircases with range $[0, \nu(+\infty, b))$ where $\nu(+\infty, b)<1$.

In addition the quantity $\nu(a, b)$ is explicitly known, see equation (7) in Appendix A. The monotonicity of $b \mapsto \nu(a, b)$ expresses the fact that stronger constraints apply to orbits (to their sojourn time in heterogeneous atoms indeed) when the coupling increases, in order to ensure that they remain bounded. 


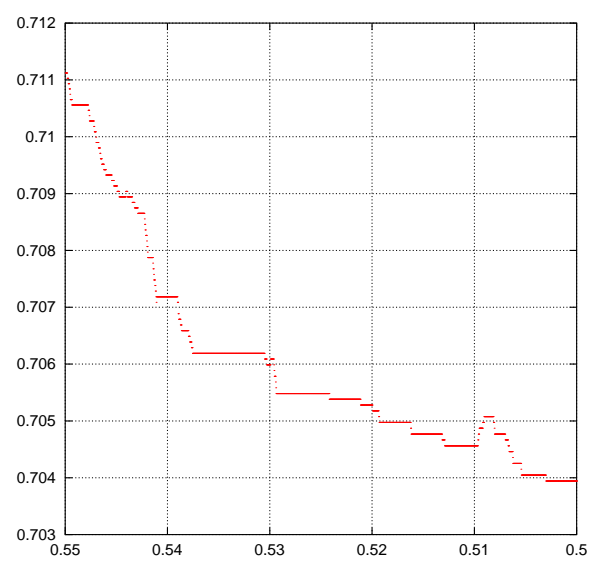

Figure 4: Entropy $\frac{\log P_{15}}{15}$ of the CML as a function of $b \in[0.5,0.55]$ for $a=2.5$ (a zoom of the intermediate curve in Figure 3). Although the curve appears to be globally decreasing, a local increase is clearly visible in the neighbourhood of 0.51 .

By using the conjugacy between the CML on its repeller $K$ and the symbolic system, together with the fact that the sets $\Omega_{\nu}$ are compact, Theorem 4.1 implies the following lower bound on the CML entropy

$$
h\left(K, F_{a, b}\right) \geqslant h\left(\Omega_{\nu(a, b)}, \sigma\right), a>2,1<b<2
$$

The properties of this bound then follow from Theorem 4.1 together with the following properties of the entropy of the family $\left\{\Omega_{\nu}\right\}_{\nu \in[0,1]}$.

Proposition 4.2. The map $\nu \mapsto h\left(\Omega_{\nu}, \sigma\right)$ defined on $[0,1]$ satisfies the following properties

- It is strictly increasing.

- It is left continuous, continuous at every irrational number and discontinuous at every rational number.

- $h\left(\Omega_{0}, \sigma\right)=\log 2, h\left(\Omega_{\nu}, \sigma\right)>\log 3$ for all $\nu>0$ and $h\left(\Omega_{1}, \sigma\right)=\log 4$.

These properties are deduced from the equation (14) for (the exponential of) $h\left(\Omega_{\nu}, \sigma\right)$ which we establish in Appendix B.

Corollary 4.3. The CML entropy lower bound $b \mapsto h\left(\Omega_{\nu(a, b)}, \sigma\right)$ (a fixed) is increasing and piecewise constant. It is left continuous, continuous at all b such that $\nu(a, b) \in \mathbb{R} \backslash \mathbb{Q}$ and discontinuous at all $b$ such that $\nu(a, b) \in \mathbb{Q}$ and $\nu\left(a, b^{\prime}\right)>\nu(a, b)$ for all $b^{\prime}>b$ (right boundary of plateaus with rational parameter).

Naturally, similar properties hold for the map $a \mapsto h\left(\Omega_{\nu(a, b)}, \sigma\right)$ ( $b$ fixed).

Based on explicit expressions of $\nu(a, b)$ and $h\left(\Omega_{\nu}, \sigma\right)$ (equations (7) in Appendix A and (14) in Appendix B respectively), the lower bound $b \mapsto h\left(\Omega_{\nu(a, b)}, \sigma\right)$ can be compared to the numerically computed entropy see Figure 5. This picture shows that the lower bound is a good approximation of the entropy which not only reflects the monotonic behaviour but also its changes in convexity.

As a final comment in this section, we point out that the bound $h\left(\Omega_{\nu(a, b)}, \sigma\right)$ is an improvement of the lower bound obtained in [FG04]. The latter reads $h\left(\Omega_{\frac{n(a, b)}{n(a, b)+1}}, \sigma\right)$ where $n(a, b)$ is the largest integer $n$ such that all sequences in $\Omega_{\frac{n}{n+1}}$ are admissible. ${ }^{6}$ The improvement is particularly significant in the neighbourhood of $b=1$ where $h\left(\Omega_{\frac{n(a, b)}{n(a, b)+1}}, \sigma\right)=\log 2$ and $h\left(\Omega_{\nu(a, b)}, \sigma\right)>\log 3$ (ie. where $0<\nu(a, b)<1 / 2$ ).

\footnotetext{
${ }^{6}$ Equivalently, the integer $n(a, b)$ can be characterized as the largest integer such that $\frac{n}{n+1} \leqslant \nu(a, b)$.
} 

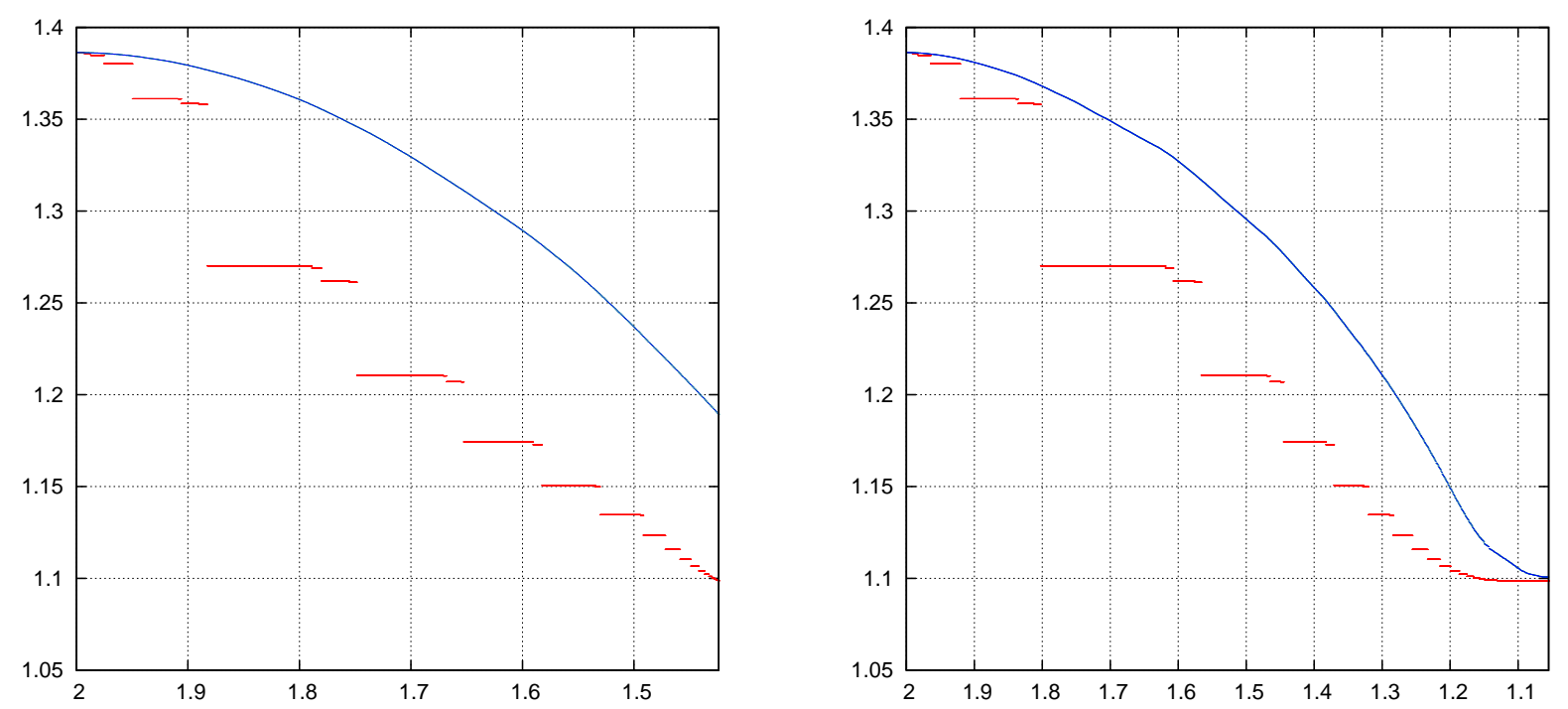

Figure 5: Plots of the entropy $\frac{\log P_{15}}{15}$ (upper/blue curve) together with the lower bounds $b \mapsto h\left(\Omega_{\nu(a, b)}, \sigma\right)$ (lower/red curve) in the domains where $\nu(a, b)>0$. Left picture: $a=2.5$. Right picture: $a=10$.

\subsection{Improving the lower bound}

As Figure 5 suggests, the lower bound is not very accurate away from the boundaries of the expanding domain. In order to improve this bound, we now show the existence of additional admissible sequences than only those in $\Omega_{\nu(a, b)}$. For the sake of simplicity, we actually focus on enlarging the sets $\Omega_{\frac{n(a, b)}{n(a, b)+1}}$.

Recall that the set $\Omega_{\frac{n}{n+1}}$ consists of sequences for which the length of every word $10^{k}$ and $01^{k}$ does not exceed $n$. Consider now the set $\Xi_{n} \supset \Omega_{\frac{n}{n+1}}(n \geqslant 1)$ of sequences such that the only possible occurrences of $01^{n+1}$ (resp. $10^{n+1}$ ) are in the words $01^{c} 01^{n+1} 1001^{k} 01^{c}\left(\right.$ resp. $10^{c} 10^{n+1} 0110^{k} 10^{c}$ ) where $0 \leqslant k \leqslant n-1$. Here $01^{c}$ denotes any symbol in $\{00,11,10\}$ and similarly for $10^{c}$.

The set $\Xi_{n}$ is an irreducible subshift of finite type which contains forbidden words of $\Omega_{\frac{n}{n+1}}$. Corollary 4.4.9 in [LM95] then implies that its entropy is larger than that of $\Omega_{\frac{n}{n+1}}$.

By using the following inequality (which holds for all $t \in \mathbb{N}$ when $b<2$ ) repeatedly

$$
\Psi_{e}\left(\theta^{0} \cdots \theta^{t-1} 1001 \theta^{t+2} \cdots\right)<\Psi_{e}\left(\theta^{0} \cdots \theta^{t-1} 1110 \theta^{t+2} \cdots\right)
$$

one constructs, for any sequence $\theta \in \Xi_{n}$ with $\theta^{0} \in\{01,00\}$, a sequence $\bar{\theta} \in \Omega_{\frac{n}{n+1}}$ with $\bar{\theta}^{0}=\theta^{0}$ such that

$$
\Psi_{e}(\theta) \leqslant \Psi_{e}(\bar{\theta})
$$

This implies that the sets $\Omega_{\frac{n}{n+1}}$ and $\Xi_{n}$ are simultaneously admissible. In other words, all sequences in $\Xi_{n(a, b)}$ are admissible and we have the following CML entropy lower bound.

Proposition 4.4. For every $a>2$ and $b \in(1,2)$ such that $n(a, b) \geqslant 1$, the following inequalities hold

$$
h\left(K, F_{a, b}\right) \geqslant h\left(\Xi_{n(a, b)}, \sigma\right)>h\left(\Omega_{\frac{n(a, b)}{n(a, b)+1}}, \sigma\right)
$$

In terms of orbits, this statement means that within the plateau $\nu(a, b)=\frac{n(a, b)}{n(a, b)+1}$, all orbits passing $n(a, b)+1$ consecutive iterations in one heterogeneous atom are also bounded provided they pass the complementary heterogeneous atom immediately afterward.

An explicit equation for $h\left(\Xi_{n}, \sigma\right)$, which allows to compare these lower bounds, is computed in Appendix C. Examples are plotted on Figure 6 which show that the family $\left\{\Xi_{n}\right\}$ provides a significant improvement of 

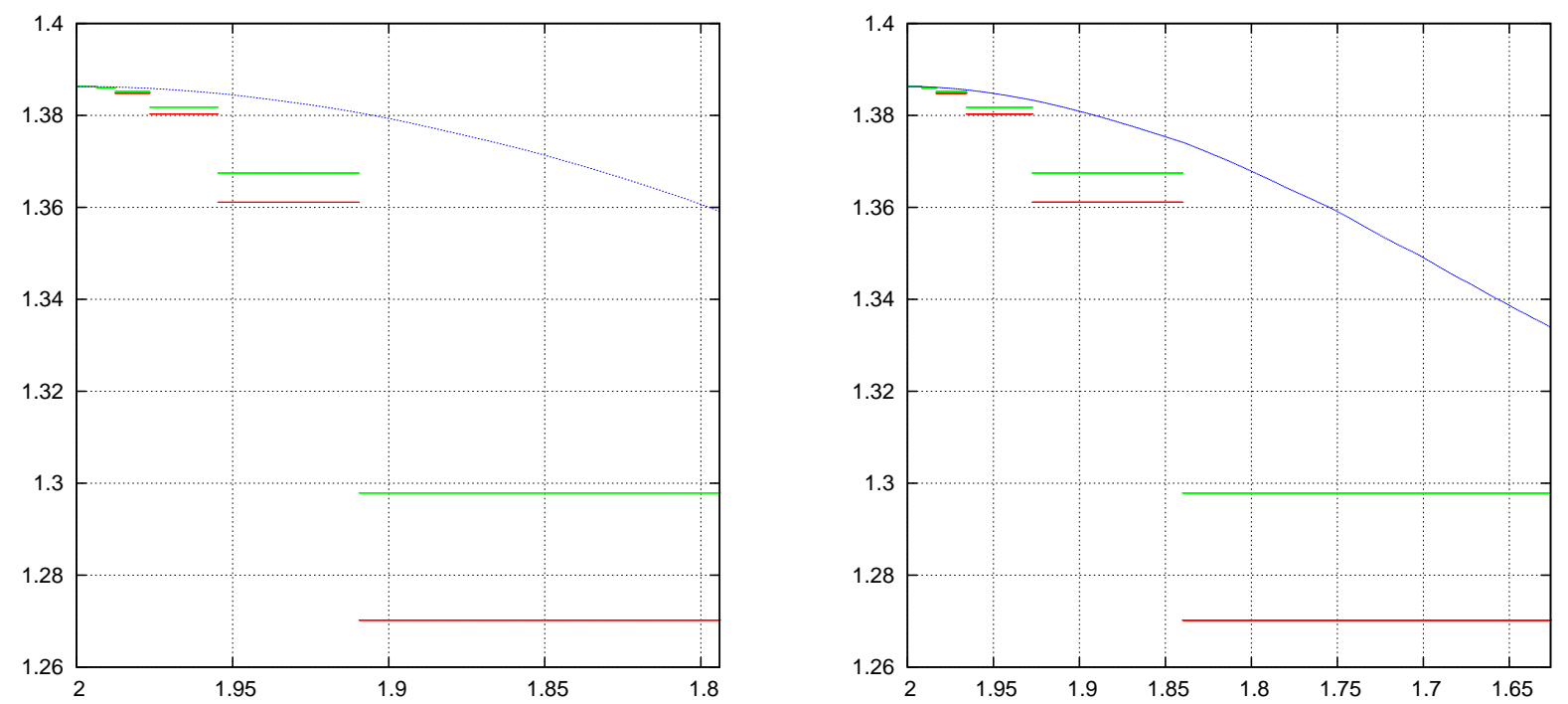

Figure 6: Plots of the entropy $\frac{\log P_{15}}{15}$ (upper/blue curve) together with the lower bounds $b \mapsto h\left(\Xi_{n(a, b)}, \sigma\right)$ (intermediate/green curve) and $b \mapsto h\left(\Omega_{\frac{n(a, b)}{n(a, b)+1}}, \sigma\right)$ (lower/red curve) in the domains where $n(a, b) \geqslant 1($ ie. $\nu(a, b) \geqslant 1 / 2)$. Left picture: $a=2.5$. Right picture: $a=10$.

the lower bound. Yet the improved lower bound is not optimal and could be further improved. For instance, one could consider the refinement which consists in allowing for the words

$$
01^{c} 01^{n+1} 1001^{n} 1001^{k} 01^{c} \text { and } 10^{c} 10^{n+1} 0110^{n} 0110^{k} 10^{c}, 1 \leqslant k \leqslant n-1
$$

\subsection{Improved bound for $a$ in the neighbourhood of 2}

\subsubsection{Behaviour of $\nu(a, b)$ at the boundaries of the domain of $a$}

As suggested in Theorem 4.1 the lower bound $b \mapsto h\left(\Omega_{\nu(a, b)}, \sigma\right)$ pointwise converges when $a \rightarrow+\infty$ to $b \mapsto h\left(\Omega_{\nu(+\infty, b)}, \sigma\right)$ whose graph is non-trivial. A flavour of this graph is given by the right picture of Figure 5 .

On the opposite Theorem 4.1 also indicates that the lower bound becomes trivial when $a \rightarrow 2$ since we have $\nu(a, b)=0$ (and then $h\left(\Omega_{\nu(a, b)}, \sigma\right)=\log 2$ ) when $a$ is close to 2 depending on $b$.

In addition for any sequence $\theta=\left\{\theta_{0}^{t} \theta_{1}^{t}\right\}_{t \in \mathbb{N}}$ such that $\theta_{0}^{0}=0$ and $\theta_{0}^{t}=1(t \geqslant 1)$ we have

$$
\Psi_{e}(\theta)=\frac{2-b}{b-1}+\sum_{k=1}^{\infty}\left(a^{-k}-b^{-k}\right) \theta_{1}^{k} \geqslant \frac{2-a}{a-1}
$$

and then $\Psi_{e}(\theta) \geqslant 0$ for every $b>1$ when $a \leqslant 2 .{ }^{7}$ It means that any such sequence is not admissible when $a \leqslant 2$. More generally, one shows that for every $a<2$ and $b>1$, there exists $n \in \mathbb{N}$ such that any sequence $\theta$ for which there exist $s$ and $t$ such that $\theta_{s}^{t}=0$ and $\theta_{s}^{t+k}=1(1 \leqslant k \leqslant n)$, is not admissible.

\subsubsection{Lower bound based on another family of sets}

In order to obtain non trivial lower bounds when $a$ is close to 2 , the previous comment suggests to consider independent constraints on each component $\left\{\theta_{s}^{t}\right\}_{t \in \mathbb{N}}$ of symbolic sequences. Accordingly, we start by

\footnotetext{
${ }^{7}$ The conjugacy between the CML on its repeller (in the expanding domain and the left shift on forward symbolic sequences extends to $a \leqslant 2$.
} 
viewing symbolic sequences $\theta=\left\{\theta_{0}^{t} \theta_{1}^{t}\right\}_{t \in \mathbb{N}}$ as being composed of two components, ie. $\theta=\left(\left\{\theta_{0}^{t}\right\}_{t \in \mathbb{N}},\left\{\theta_{1}^{t}\right\}_{t \in \mathbb{N}}\right)$. Obviously each component is a sequence in $\{0,1\}^{\mathbb{N}}$.

The following constraints are applied to components. Given $\nu \in\left[\frac{1}{2}, 1\right]$, let $\lambda_{\nu} \subset\{0,1\}^{\mathbb{N}}$ defined as the set of sequences $\left\{\vartheta^{t}\right\}_{t \in \mathbb{N}}$ such that

$$
\left\lfloor\left(t_{2}-t_{1}\right)(1-\nu)\right\rfloor \leqslant \sum_{t=t_{1}}^{t_{2}-1} \vartheta^{t} \leqslant\left\lceil\left(t_{2}-t_{1}\right) \nu\right\rceil \quad \forall t_{1}<t_{2} \in \mathbb{N}
$$

In other words, every word of length $k$ in $\lambda_{\nu}$ contains at most $\lceil k \nu\rceil$ symbols 0 's and at most $\lceil k \nu\rceil$ symbols 1's. Now considering independent constraints on the components means that we consider the product $\Lambda_{\nu}:=\lambda_{\nu} \times \lambda_{\nu} \subset\{01,00,11,10\}^{\mathbb{N}}$.

Proceeding similarly as for the proof of Theorem 4.1, one obtains the maximum value of $\nu$, depending on $a$ and $b$, such that all sequences in $\Lambda_{\nu}$ satisfy the admissibility condition (3). (For the proof see Appendix D.)

Theorem 4.5. For every $a>2$ and $1<b<2$, there exists $\nu_{\Lambda}(a, b) \in\left[\frac{1}{2}, 1\right)$ such that for every $\frac{1}{2} \leqslant \nu<$ $\nu_{\Lambda}(a, b)$ every sequence in $\Lambda_{\nu}$ is the code of some bounded orbit. Moreover, for every $\nu>\nu_{\Lambda}(a, b)$ there are sequences in $\Lambda_{\nu}$ not corresponding to any bounded orbit code.

The map $b \mapsto \nu_{\Lambda}(a, b)$ is an increasing Devil's staircase with range $\left[\frac{1}{2}, 1\right)$.

The map $a \mapsto \nu_{\Lambda}(a, b)$ is an increasing Devil's staircase with range the following behaviour $\lim _{a \rightarrow 2^{+}} \nu_{\Lambda}(a, b)>\frac{1}{2}$ whenever $b^{*} \leqslant b<2$ where the quantity $b^{*}>1$ is explicitly known.

In short terms, considering the family $\left\{\Lambda_{\nu}\right\}$ ensures that the CML bounded dynamics does not reduce to the diagonal when $a$ is close to 2, provided that $b$ is not too small.

As for the inequality (4), Theorem 4.5 implies the following lower bound

$$
h\left(K, F_{a, b}\right) \geqslant h\left(\Lambda_{\nu_{\Lambda}(a, b)}, \sigma\right), a>2,1<b<2
$$

Moreover, the family $\left\{\Lambda_{\nu}\right\}_{\nu \in\left[\frac{1}{2}, 1\right]}$ has similar entropy properties to those in Proposition 4.2. These follow from an explicit equation for the entropy of $\lambda_{\nu}$, see Appendix D.

Proposition 4.6. The map $\nu \mapsto h\left(\Lambda_{\nu}, \sigma\right)$ defined on $\left[\frac{1}{2}, 1\right]$ satisfies the following properties

- It is strictly increasing.

- It is left continuous, continuous at every irrational number and discontinuous at every rational number.

- $h\left(\Lambda_{\frac{1}{2}}, \sigma\right)=\log 2$ and $h\left(\Lambda_{1}, \sigma\right)=\log 4$.

By combining the previous results, we obtain the following conclusions. (For the proof see again Appendix D.)

Corollary 4.7. The CML entropy lower bound $b \mapsto h\left(\Lambda_{\nu_{\Lambda}(a, b)}, \sigma\right)$ (a fixed) has the same properties as the map $b \mapsto h\left(\Omega_{\nu(a, b)}, \sigma\right)$.

In addition, if a is sufficiently close to 2 , we have

$$
\begin{aligned}
& h\left(\Lambda_{\nu_{\Lambda}(a, b)}, \sigma\right)=h\left(\Omega_{\nu(a, b)}, \sigma\right)=\log 2 \quad \text { if } \quad 1<b \leqslant b_{0}(a) \\
& h\left(\Lambda_{\nu_{\Lambda}(a, b)}, \sigma\right)>h\left(\Omega_{\nu(a, b)}, \sigma\right)=\log 2 \quad \text { if } \quad b_{0}(a)<b \leqslant b_{1}(a)
\end{aligned}
$$

where $1<b_{0}(a)<b_{1}(a)<2$ have the following property $\lim _{a \rightarrow 2^{+}} b_{0}(a)<\lim _{a \rightarrow 2^{+}} b_{1}(a)=2$.

In other words, the bound $h\left(\Lambda_{\nu_{\Lambda}(a, b)}, \sigma\right)$ improves the bound $h\left(\Omega_{\nu(a, b)}, \sigma\right)$ on every interval $\left[b_{0}(2+0)+\delta, 2-\delta\right]$ when $a$ is sufficiently close to 2 (depending on $\delta$ ). Moreover, since we have $n(a, b)=\nu(a, b)=0$ when $b \in\left(1, b_{1}(a)\right)$, this bound is also complementary to the bound $h\left(\Xi_{n(a, b)}, \sigma\right)$ of the previous section. 


\section{Symbolic dynamics in the strong coupling domain}

In the strong coupling domain (ie. when $0<b<1$ ) the CML is no longer expanding and the symbolic dynamics associated with its maximal hyperbolic set consists of doubly infinite sequences $\theta=\left\{\theta^{t}\right\} \in\{01,00,11,10\}^{\mathbb{Z}}$ which satisfy the admissibility condition $\left(3^{\prime}\right)$.

\subsection{Possibly admissible symbolic sequences}

A preliminary result is that the condition $\left(3^{\prime}\right)$ imposes strong restrictions on symbolic sequences, independently of parameters. Let $\mathcal{P}$ be the subset of $\{01,00,11,10\}^{\mathbb{Z}}$ of sequences $\theta$ such that

- either $\theta$ is homogeneous

- or $\theta$ has infinitely many heterogeneous symbols in the past (and finitely or infinitely many in the future). The heterogeneous symbols need not to be consecutive but they must alternate. In other words, for any $t$ such that $\theta^{t} \in\{01,10\}$ there exists $t^{\prime}<t$ such that $\theta^{t^{\prime}}=1-\theta^{t} \in\{01,10\}$ and $\theta^{k} \in\{00,11\}$ for all $t^{\prime}<k<t$.

In particular, the sequence defined by $\theta^{2 t+s}=R^{s}(10), s \in\{0,1\} t \in \mathbb{Z}$, belongs to $\mathcal{P}$. It corresponds to the 2-periodic heterogeneous orbit which exists for every $a>2$ and $b>0 .{ }^{8}$

Let $\overline{\mathcal{P}}$ be the closure of $\mathcal{P}$ (product topology). The dynamical system $(\overline{\mathcal{P}}, \sigma)$ is a sofic shift [LM95] whose graph is given on Figure 7. The announced restrictions, which apply globally in the strong coupling domain,

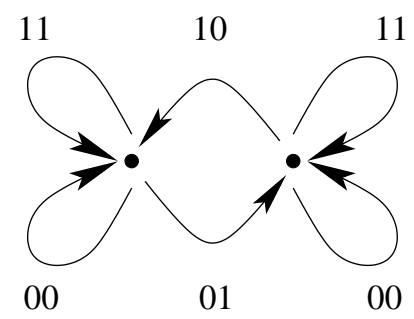

Figure 7: Graph associated with the sofic shift $(\overline{\mathcal{P}}, \sigma)$.

are given in the following statement.

Proposition 5.1. For every $a>2$ and $0<b<1$, every sequence satisfying $\left(3^{\prime}\right)$ belongs $\mathcal{P}$.

For the CML, this result provides a necessary condition for an orbit off diagonal to be bounded: the orbit must visit alternatively the two heterogeneous atoms. Moreover, it shows that the orbit of every point in the unstable manifold of the diagonal must be unbounded.

Proposition 5.1 implies that the CML entropy is bounded above by $h(\mathcal{P}, \sigma)$. By computing the largest eigenvalue of the adjency matrix (see eg. Theorem 4.3.3 in [LM95])

$$
\left(\begin{array}{ll}
2 & 1 \\
1 & 2
\end{array}\right)
$$

associated with the graph of Figure 7 , one obtains that $h(\mathcal{P}, \sigma) \leqslant h(\overline{\mathcal{P}}, \sigma)=\log 3 \simeq 1.1$. The numerically computed entropy on Figure 3 shows that this upper bound is sharp. We shall prove below that it becomes exact in the limit $a \rightarrow \infty$.

Proof of Proposition 5.1: We show that every sequence not in $\mathcal{P}$ cannot be admissible. Consider firstly the case where a nonhomogeneous sequence has finitely many heterogeneous symbols in the past. Up to symmetries $\sigma$ and $R$ we may assume that $\theta^{0}=10$ and $\theta^{t} \in\{00,11\}$ for all $t<0$.

\footnotetext{
${ }^{8}$ Indeed for such sequence we have $\Psi_{h}(\theta)=D_{h}(\theta)=\frac{b}{1+b}>0$ which implies that the condition $\theta_{s}^{t}=H \circ \Psi_{h}\left(\sigma^{t} R^{s} \theta\right)$ holds for $t=s=0$. By symmetries $\sigma$ and $R$, it holds for all $t \in \mathbb{Z}$ and $s \in\{0,1\}$.
} 
Then we have $D_{h}(\theta)=-D_{h}(R \theta)=0$. Therefore, either $S(\theta)=\Psi_{h}(\theta)<0$ and the admissibility condition does not hold, or $S(\theta) \geqslant 0$. In the latter case, the admissibility does neither hold because $(R \theta)^{0}=01$ and $\Psi_{h}(R \theta)=S(R \theta)=S(\theta) \geqslant 0$.

We now show the heterogeneous symbols must alternate. By contradiction assume that $\theta^{0}=10$ and there exists $t^{\prime}<0$ such that $\theta^{t^{\prime}}=10$ and $\theta^{t} \in\{00,11\}$ for all $t^{\prime}<t<0$. If $D_{h}(\theta)<0$, then we are done. Indeed, either $S(\theta)<0$ and $\Psi_{h}(\theta)<0$ or $S(\theta) \geqslant 0$ and thus $\Psi_{h}(R \theta) \geqslant 0$. In both cases the condition $\left(3^{\prime}\right)$ does not hold.

In the case where $D_{h}(\theta) \geqslant 0$, there must be $t^{\prime \prime}<t^{\prime}$ such that $\theta^{t^{\prime \prime}}=01$. Indeed if otherwise $\theta^{t} \in\{00,11,10\}$ for all $t<0$, then we would have $D_{h}(\theta)<0$ (using also that $\theta^{t^{\prime}}=10$ ). Assume that $t^{\prime \prime}$ is the largest such integer, ie. $\theta^{t} \in\{00,11,10\}$ for all $t^{\prime \prime}<t<0$.

The conditions $D_{h}(\theta) \geqslant 0$ and $\theta^{t} \in\{00,11,10\}$ for all $t^{\prime \prime}<t<0$ imply that $D_{h}\left(\sigma^{t^{\prime \prime}} \theta\right)>0$. If $S\left(\sigma^{t^{\prime \prime}} \theta\right) \geqslant 0$ then we have $\Psi_{h}\left(\sigma^{t^{\prime \prime}} \theta\right) \geqslant 0$. Since $\left(\sigma^{t^{\prime \prime}} \theta\right)^{0}=01$, the condition $\left(3^{\prime}\right)$ cannot hold. Otherwise $S\left(\sigma^{t^{\prime \prime}} \theta\right)<0$ and then $\Psi_{h}\left(\sigma^{t^{\prime \prime}} R \theta\right)<0$ whence $\left(\sigma^{t^{\prime \prime}} R \theta\right)^{0}=10$. The condition $\left(3^{\prime}\right)$ can neither hold in this case. Therefore the heterogeneous symbols must alternate and the proof is complete.

\subsection{Bounds in the lower part of the strong coupling domain}

With a set of possibly admissible sequences given, we proceed analogously to as in the weak coupling domain to characterize the dynamics for strong coupling. The analysis splits into two parts: a lower part for which upper and lower bounds are obtained, and an upper part (the synchronized regime) for which all admissible sequences are determined.

\subsubsection{Upper bound}

The study of properties of $\Psi_{h}$ for sequences in $\mathcal{P}$ provide additional restrictions on admissible sequences which depend on parameters. These restrictions state that only sequences with short homogeneous words can be admissible, unless these words are followed by long heterogeneous ones.

Proposition 5.2. For every $a>2$ and $0<b<1$ and for every $i \in \mathbb{N}^{*}$ there exists $n(a, b, i) \in \mathbb{N}^{*}$ such that any sequence containing a homogeneous word of length $n(a, b, i)$ followed by a heterogeneous word of length $i$ or smaller, is not admissible.

Moreover, the map $i \mapsto n(a, b, i)$ is increasing and we have $\lim _{b \rightarrow 1} n(a, b, i)=\infty$ and $\lim _{b \rightarrow 0} n(a, b, i)=0$.

This result provides another necessary condition for an orbit off diagonal to be bounded. An orbit passing a large number of (consecutive) steps in homogeneous atoms, must either converges to the diagonal or pass a large number of steps in the heterogeneous atoms immediately afterward. In particular, any bounded orbit not in the stable manifold of the diagonal but accumulating to it, must also accumulate to the 2-periodic heterogeneous orbit.

Proof: By symmetries, we can assume without loss of generality that a sequence $\theta \in \mathcal{P}$ containing a heterogeneous word of length $i$ satisfies the following condition

$$
\theta^{t}=\left\{\begin{array}{lll}
10 & \text { if } & 0 \leqslant t \leqslant i-1 \text { and } t \text { is even } \\
01 & \text { if } & 0<t \leqslant i-1 \text { and } t \text { is odd } \\
00 & \text { if } & t=i
\end{array}\right.
$$

For such a sequence, we have $S(\theta) \leqslant-a^{-i}+\sum_{k=i+1}^{\infty} a^{-k}=-a^{-i} \frac{a-2}{a-1}<0$ (notice that the bound is attained if $\theta^{t}=11$ for all $\left.t \geqslant i+1\right)$.

Let now $n(a, b, i)$ be the smallest integer such that

$$
b^{n(a, b, i)+1} \leqslant a^{-i} \frac{a-2}{a-1}
$$

Notice that the map $i \mapsto n(a, b, i)$ is increasing and that we have $\lim _{b \rightarrow 1^{-}} n(a, b, i)=+\infty$ and $\lim _{b \rightarrow 0^{+}} n(a, b, i)=0$. 
Now if the previous sequence $\theta$ also satisfies the condition

$$
\theta^{t} \in\{00,11\}, \forall-n(a, b, i) \leqslant t \leqslant-1
$$

then we have $\left|D_{h}(\theta)\right|=\left|\sum_{k=n(a, b, i)+1}^{\infty} b^{k}\left(\theta_{1}^{-k}-\theta_{0}^{-k}\right)\right|<b^{n(a, b, i)+1}$, because heterogeneous symbols alternate for every sequence $\theta \in \mathcal{P}$. Consequently $\Psi_{h}(\theta)=S(\theta)+D_{h}(\theta)<0$ and $\theta^{0}=10$. The admissibility condition cannot hold.

\subsubsection{Lower bound}

Proposition 5.2 indicates that the nonadmissibility of a nonhomogeneous sequence might depend on the length of its homogeneous words. We now show that this length can actually acts as an admissibility criterion, at least when $a$ is sufficiently large.

In order to obtain a lower bound on admissible sequences, we start by introducing a family $\left\{\Gamma_{n}\right\}_{n \in \mathbb{N}^{*}}$ of sets included in $\mathcal{P}$ and defined as follows.

- All homogeneous sequences belong to every $\Gamma_{n}$.

- A nonhomogeneous sequence belongs to $\Gamma_{n}$ iff all its homogeneous words have length not exceeding $n$.

The map $n \mapsto h\left(\Gamma_{n}, \sigma\right)$ is strictly increasing and we have $h\left(\Gamma_{1}, \sigma\right)=\log 2$ and $\lim _{n \rightarrow+\infty} h\left(\Gamma_{n}, \sigma\right)=\log 3$, see Appendix E.

Proposition 5.3. For every $a>5$ and $0<b<1$ there exists $n^{\prime}(a, b) \in \mathbb{N}^{*}$ such that the set of admissible sequences contains $\Gamma_{n^{\prime}(a, b)}$. In addition for any $n>n^{\prime}(a, b)$ there are sequences in $\Gamma_{n}$ not corresponding to any bounded code.

The following CML entropy lower bounds holds $h\left(K, F_{a, b}\right) \geqslant h\left(\Gamma_{n^{\prime}(a, b)}, \sigma\right)$ and the mapping $b \mapsto n^{\prime}(a, b)$ is increasing. Moreover it has the following limit $2 \leqslant \lim _{b \rightarrow 1^{-}} n^{\prime}(a, b)<+\infty$ for every $a>5$.

The mapping $a \mapsto n^{\prime}(a, b)$ is increasing and we have $\lim _{a \rightarrow+\infty} n^{\prime}(a, b)=+\infty$ for every $0<b<1$.

As a consequence the mapping $b \mapsto h\left(\Gamma_{n^{\prime}(a, b)}, \sigma\right)$ is an increasing step function (with a finite number of steps) which pointwise converges to the constant mapping $b \mapsto \log 3$ when $a \rightarrow+\infty$. The property $\lim _{b \rightarrow 1^{-}} n^{\prime}(a, b) \geqslant 2$ ensures that this lower bound is not trivial (ie. $\left.h\left(\Gamma_{n^{\prime}(a, b)}, \sigma\right)>\log 2\right)$ when $b$ is close to 1 .

The proof of this Proposition is given in Appendix E. Notice that the condition $a>5$ (which ensures that $\left.\lim _{b \rightarrow 1^{-}} n^{\prime}(a, b) \geqslant 2\right)$ is not optimal. The proof actually shows that a sufficient condition is $a^{3}-4 a^{2}-4 a-1>0$ which holds for $a>4.865$. In addition and together with Proposition 5.1, the Proposition implies that

$$
\lim _{a \rightarrow+\infty} h\left(K, F_{a, b}\right)=\log 3, \forall 0<b<1 .
$$

By using the explicit expression of $n^{\prime}(a, b)$ and the equation for the entropy of $\left(\Gamma_{n}, \sigma\right)$, the bound $h\left(\Gamma_{n^{\prime}}(a, b), \sigma\right)$ can be computed explicitly and compared to the numerically computed entropy. The results are presented on Figure 8 which shows a good agreement between the two quantities and better agreement for stronger values of $a$. Finally, we mention that, as for weak couplings, the lower bound can be improved by considering a continuous family of sets $\left\{\Gamma_{\nu}\right\}$ instead of a discrete one.

\subsection{Synchronisation}

We conclude our study of symbolic dynamics over the coupling parameter range by the neighbourhood of $b=0$. In correspondence to the uncoupled regime where the CML $F_{a, b}$ is topologically conjugated to the uncoupled system $F_{a, a}$, in this domain, the CML turns out to be topologically conjugated to the fully coupled system $F_{a, 0}$. It means that all bounded orbits lie on the diagonal excepted for the 2-periodic heterogeneous orbit. In particular, the entropy equals $\log 2$. By analogy with the fact that in strongly coupled smooth systems all orbits asymptotically lie on the diagonal [PRK01], this property is called synchronisation. Technically speaking, it can be formulated as follows. 

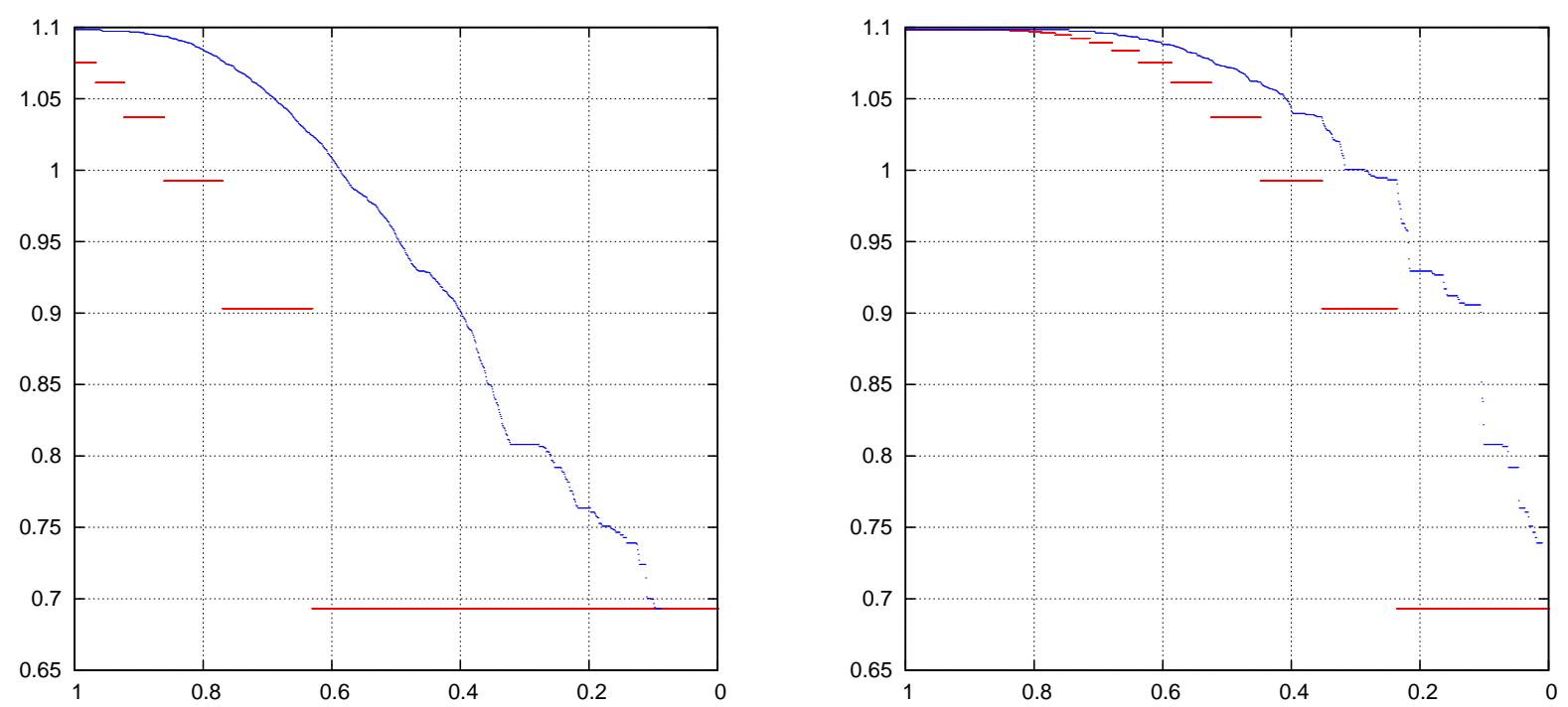

Figure 8: Plots of the entropy $\frac{\log P_{15}}{15}$ (upper/blue curve) together with the lower bound $b \mapsto h\left(\Gamma_{n(a, b)}, \sigma\right)$ (lower/red curve) in the strong coupling domain. Left picture: $a=10$. Right picture: $a=100$.

Proposition 5.4. For every $a>2$ there exists $b_{\text {sync }}(a)>0$ such that when $0<b<b_{\text {sync }}(a)$, a symbolic sequence is the code of a bounded orbit iff it belongs to $\Gamma_{0}$.

Proof: If a sequence $\theta \in \mathcal{P}$ does not belong to $\Gamma_{0}$, then there must be $t \in \mathbb{Z}$ such that $\theta^{t} \in\{01,10\}$ and $\theta^{t+1} \in\{00,11\}$. By symmetries, we may assume without loss of generality that $\theta^{0}=10$ and $\theta^{1}=00$.

This corresponds to assuming that $i=1$ in the beginning of the proof of Proposition 5.2. Hence we have $S(\theta) \leqslant-a^{-1} \frac{a-2}{a-1}$.

On the other hand, according to monotonicity of $D_{h}$ with respect to the lexicographic ordering on backward sequences (see proof of Proposition 5.3) we have $D_{h}(\theta) \leqslant D_{h}\left({ }^{\infty} 1101\right)=b$.

Consequently when $b<b_{\text {sync }}(a):=a^{-1} \frac{a-2}{a-1}$, we have $\Psi_{h}(\theta)<0$ and then $\theta$ does not satisfies $\left(3^{\prime}\right)$. This implies that only those sequences in $\Gamma_{0}$ can be admissible. Since all of them do, the proposition follows.

The quantity $b_{\text {sync }}(a)=\frac{a-2}{a(a-1)}$ obtained in the proof is compared to the numerically computed synchronisation threshold, see Table 1. This table shows a good agreement between the two quantities, which is better for larger $a$.

\begin{tabular}{|c|c|c|}
\hline$a$ & $b_{\text {num }}(a)$ & $b_{\text {sync }}(a)$ \\
\hline 2.1 & 0.236 & 0.043 \\
2.5 & 0.187 & 0.133 \\
5 & 0.176 & 0.150 \\
10 & 0.097 & 0.089 \\
100 & 0.0099 & 0.0099 \\
\hline
\end{tabular}

Table 1: Table of numerically computed synchronisation threshold $b_{\text {num }}(a)$ together with the corresponding lower bound $b_{\text {sync }}(a)$. 


\section{A Proof of Theorem 4.1}

By using the symmetry $\theta \mapsto 1-\theta$, translation invariance and compactness of $\Omega_{\nu}$, one shows that all sequences in $\Omega_{\nu}$ satisfy the admissibility condition (3) iff

$$
\sup _{\theta \in \Omega_{\nu}^{0}} \Psi_{e}(\theta)<0
$$

where $\Omega_{\nu}^{0}$ is the set of sequences in $\Omega_{\nu}$ such that $\theta_{0}^{0}=0$.

The proof consists in obtaining an expression for $\sup _{\theta \in \Omega^{0}} \Psi_{e}(\theta)$ which is simple enough to be studied. We proceed in two steps. Firstly we prove that this supremum is attained in the subset of $\Omega_{\nu}^{0}$ composed of sequences such that $\theta^{t} \in\{11,10\}$ for all $t \geqslant 1$. Secondly we show that the supremum on this subset is attained as a maximum over a finite set. The first step is given in the next statement.

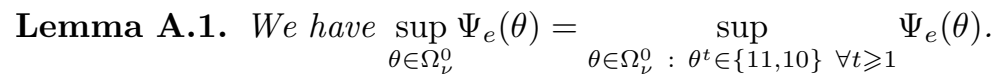

Proof: Given a sequence $\theta \in\{01,00,11,10\}^{\mathbb{N}}$, consider the following quantities

$$
t_{01}(\theta)=\inf \left\{t \geqslant 1: \theta^{t}=01\right\}, t_{10}(\theta)=\inf \left\{t>t_{01}(\theta): \theta^{t}=10\right\}
$$

and

$$
t_{01}^{(2)}(\theta)=\sup \left\{t_{01}(\theta) \leqslant t \leqslant t_{10}(\theta): \theta^{t}=01\right\}
$$

In particular, if $t_{01}^{(2)}(\theta)<+\infty$ then we have $\theta^{t} \in\{00,11\}$ for all $t_{01}^{(2)}(\theta)<t<t_{10}(\theta)$.

Consider the mapping $P$ defined in $\{01,00,11,10\}^{\mathbb{N}}$ as follows. If $t_{01}(\theta)+t_{10}(\theta)=+\infty$, then $P(\theta)=\theta$. Otherwise we set

$$
P(\theta)^{t}= \begin{cases}\theta^{t} & \text { if } \quad t<t_{01}^{(2)}(\theta) \text { or } t>t_{10}(\theta) \\ 11 & \text { if } \quad t_{01}^{(2)}(\theta) \leqslant t \leqslant t_{10}(\theta)\end{cases}
$$

It is simple to check that $P$ has the following properties which hold for any sequence $\theta \in\{01,00,11,10\}^{\mathbb{N}}$

- If $\theta \in \Omega_{\nu}^{0}$, then $P(\theta) \in \Omega_{\nu}^{0}$.

- $\Psi_{e}(\theta) \leqslant \Psi_{e} \circ P(\theta)$.

- $t_{01} \circ P(\theta) \geqslant t_{01}(\theta)$.

- $t_{10} \circ P(\theta)>t_{10}(\theta)$ if $t_{10}(\theta)<+\infty$

- The limit $\lim _{n \rightarrow \infty} P^{n}(\theta)=: P^{\infty}(\theta)$ exists.

In addition, we have $t_{10} \circ P^{\infty}(\theta)=+\infty$ for all $\theta$ which implies

$$
P^{\infty}(\theta)^{t} \in\left\{\begin{array}{lll}
\{00,11,10\} & \text { if } \quad 1 \leqslant t<t_{01} \circ P^{\infty}(\theta) \\
\{01,00,11\} & \text { if } t \geqslant t_{01} \circ P^{\infty}(\theta)
\end{array}\right.
$$

Let now the mapping $Q$ defined in $\{01,00,11,10\}^{\mathbb{N}}$ by

$$
Q(\theta)^{t}= \begin{cases}\theta^{t} & \text { if } t=0, \theta^{t}=11 \text { or } \theta^{t}=10 \\ 11 & \text { if } t \geqslant 1 \text { and }\left(\theta^{t}=01 \text { or } \theta^{t}=00\right)\end{cases}
$$

The mapping $Q$ satisfies the following properties

- If $\theta \in \Omega_{\nu}^{0}$ and $t_{10}(\theta)=+\infty$, then $Q(\theta) \in \Omega_{\nu}^{0}$.

- $\Psi_{e}(\theta) \leqslant \Psi \circ Q(\theta)$.

- $Q(\theta)^{t} \in\{11,10\}$ for all $t \geqslant 1$. 
Collecting the results, we conclude that

- $Q \circ P^{\infty}\left(\Omega_{\nu}^{0}\right) \subset \Omega_{\nu}^{0}$,

- $\Psi_{e}(\theta) \leqslant \Psi_{e} \circ Q \circ P^{\infty}(\theta)$,

- $Q \circ P^{\infty}(\theta)^{t} \in\{11,10\}$ for all $t \geqslant 1$

from which the Lemma follows.

In order to restrict further the set where to maximise $\Psi_{e}$, it is convenient to rewrite this function restricted to sequences in $\Omega_{\nu}^{0}$ such that $\theta^{t} \in\{11,10\}$ for all $t \geqslant 1$ - under the following form

$$
\Psi_{e}(\theta)=\frac{2-a}{a-1}+\Phi(J)
$$

where

- the sequence $J:=J(\theta)=\left\{J_{k}\right\}_{k \in \mathbb{N}}$ is defined by $J_{0}=0$ and $J_{k}=\sum_{t=1}^{k}\left(\theta_{0}^{t}-\theta_{1}^{t}\right)=\sum_{t=1}^{k}\left(1-\theta_{1}^{t}\right)$ for $k \geqslant 1$

- and $\Phi(J):=\sum_{k=1}^{+\infty}\left(\frac{b-1}{b^{k+1}}-\frac{a-1}{a^{k+1}}\right) J_{k}$.

We have

$$
\sup _{\theta \in \Omega_{\nu}^{0}} \Psi_{e}(\theta)=\frac{2-a}{a-1}+\sup _{J \in \Theta_{\nu}} \Phi(J)
$$

where the set $\Theta_{\nu}(\nu \in[0,1])$ is defined by

$$
\Theta_{\nu}=\left\{\left\{J_{k}\right\}_{k \in \mathbb{N}} \in \mathbb{N}^{\mathbb{N}}: J_{0}=0, J_{k} \leqslant J_{k+1}, J_{k_{2}}-J_{k_{1}} \leqslant\left\lceil\left(k_{2}-k_{1}\right) \nu\right\rceil \forall 0 \leqslant k_{1}<k_{2}\right\}
$$

Since $1<b<a$, there exists an integer $k^{*} \geqslant 1$ which depends on $a$ and $b$ such that

$$
\begin{cases}\frac{b-1}{b^{k+1}}-\frac{a-1}{a^{k+1}}<0 & \text { if } \quad 0 \leqslant k<k^{*} \\ \frac{b-1}{b^{k+1}}-\frac{a-1}{a^{k+1}} \geqslant 0 & \text { if } \quad k \geqslant k^{*}\end{cases}
$$

We have $k^{*}=1$ when $b$ is close enough to 2 (depending on $a$ ) and $k^{*} \rightarrow \infty$ when $b \rightarrow 1$.

Consider finally the function $\phi_{\nu}(\alpha)=\sum_{k=1}^{+\infty}\left(\frac{b-1}{b^{k+1}}-\frac{a-1}{a^{k+1}}\right)\lceil k \nu-\alpha\rceil$. The desired simple expression of the supremum is given by the following statement. (The notation $\lfloor\cdot\rfloor$ stands for the floor function.)

Lemma A.2. We have $\sup _{J \in \Theta_{\nu}} \Phi(J)=\eta(\nu):=\max _{0 \leqslant k<k^{*}} \phi_{\nu}(k \nu-\lfloor k \nu\rfloor)$.

Proof: The proof consists of two steps. 1) We show that $\sup _{J \in \Theta_{\nu}} \Phi(J)=\sup _{\alpha \in[0,1)} \phi_{\nu}(\alpha)$. 2) We show that $\sup _{\alpha \in[0,1)} \phi_{\nu}(\alpha)=\max _{0 \leqslant k<k^{*}} \phi_{\nu}(k \nu-\lfloor k \nu\rfloor)$.

1) Given $J \in \Theta_{\nu}$, let $\underline{\alpha}=\inf \left\{\alpha>0: J_{k} \geqslant\lceil k \nu-\alpha\rceil, 0 \leqslant k<k^{*}\right\}<\infty$. We show that $\Phi(J) \leqslant \phi_{\nu}(\underline{\alpha}-\lfloor\underline{\alpha}\rfloor)$ from which the desired conclusion immediately follows. (Indeed, any sequence $J_{k}=\lceil k \nu-\alpha\rceil$ with $\lfloor\alpha\rfloor=0$ belongs to $\Theta_{\nu}$ and is such that $\Phi(J)=\phi_{\nu}(\alpha)$ ).

(a) We have $J_{k} \geqslant\lceil k \nu-\alpha\rceil$ for $0 \leqslant k<k^{*}$ whenever $\alpha>\underline{\alpha}$. By taking the right limit $\alpha \rightarrow \underline{\alpha}^{+}$, it results that $J_{k} \geqslant\lceil k \nu-\underline{\alpha}\rceil$ for $0 \leqslant k<k^{*}$.

(b) We claim that there exists $k_{0} \in\left\{0, \cdots, k^{*}-1\right\}$ such that $J_{k_{0}}=k_{0} \nu-\underline{\alpha}$. Indeed, if $\underline{\alpha}=0$ then this equality holds for $k_{0}=0$. If otherwise $\underline{\alpha}>0$, by contradiction assume that such $k_{0}$ does not exist. Then one can find $0<\alpha<\underline{\alpha}$ such that $\lceil k \nu-\alpha\rceil=\lceil k \nu-\underline{\alpha}\rceil$ for all $k \in\left\{0, \cdots, k^{*}-1\right\}$. But this is impossible by definition of $\underline{\alpha}$. 
(c) We claim that $J_{k} \leqslant\lceil k \nu-\underline{\alpha}\rceil$ for all $k \geqslant k^{*}$. By contradiction, assume the existence of $k_{1}>k^{*}$ such that $J_{k_{1}}>\left\lceil k_{1} \nu-\underline{\alpha}\right\rceil$. Then by (b), we have $J_{k_{1}}-J_{k_{0}}>\left\lceil k_{1} \nu-\underline{\alpha}\right\rceil-\left(k_{0} \nu-\underline{\alpha}\right)=\left\lceil\left(k_{1}-k_{0}\right) \nu\right\rceil$ which contradicts the inequality $J_{k_{1}}-J_{k_{0}} \leqslant\left\lceil\left(k_{1}-k_{0}\right) \nu\right\rceil$ in the definition of $\Theta_{\nu}$.

Finally, the properties (a) and (c) imply that

$$
\Phi(J) \leqslant \sum_{k=1}^{+\infty}\left(\frac{b-1}{b^{k+1}}-\frac{a-1}{a^{k+1}}\right)\lceil k \nu-\underline{\alpha}\rceil=\phi_{\nu}(\underline{\alpha}) \leqslant \phi_{\nu}(\underline{\alpha}-\lfloor\underline{\alpha}\rfloor)
$$

2) If $k^{*}=1$ then $\phi_{\nu}$ is decreasing on $[0,1)$ and the claim follows immediately. Assume then that $k^{*}>1$ and consider the decomposition $\phi_{\nu}=\phi_{-}+\phi_{+}$where

$$
\phi_{-}(\alpha)=\sum_{k=1}^{k^{*}-1}\left(\frac{b-1}{b^{k+1}}-\frac{a-1}{a^{k+1}}\right)\lceil k \nu-\alpha\rceil, \alpha \in[0,1)
$$

By definition of $k^{*}$, the map $\alpha \mapsto \phi_{-}(\alpha)$ is an increasing right continuous step function with discontinuities at $k \nu-\lfloor k \nu\rfloor$ for $0 \leqslant k<k^{*}$. Moreover, the map $\alpha \mapsto \phi_{+}(\alpha)$ is decreasing (strictly if $\nu \in \mathbb{R} \backslash \mathbb{Q}$ ) and right continuous. Therefore, it results that, if $k_{1} \nu-\left\lfloor k_{1} \nu\right\rfloor<k_{2} \nu-\left\lfloor k_{2} \nu\right\rfloor$ are two consecutive discontinuity points of $\phi_{-}$, we obtain that $\quad \sup \quad \phi_{\nu}(\alpha)=\phi_{\nu}\left(k_{1} \nu-\left\lfloor k_{1} \nu\right\rfloor\right)$ from which the property 2) easily follows.

Lemma A.2 shows that the admissibility of the sets $\Omega_{\nu}$ is governed by the function $\eta$. This function has the following properties (whose proof is postponed below).

Lemma A.3. The map $\nu \mapsto \eta(\nu)$ is strictly increasing, continuous et every irrational number and discontinuous at every rational number.

From relation (6) and the two lemmas, we conclude that the function $\nu(a, b)$ in Theorem 4.1 is given by (the dependence on $a$ and on $b$ has been added to $\eta$ )

$$
\nu(a, b)=\sup \left\{\nu \in[0,1]: \eta(a, b, \nu) \leqslant \frac{a-2}{a-1}\right\}
$$

Indeed, for any $\nu<\nu(a, b)$, we have $\eta(a, b, \nu)<\eta(a, b, \nu(a, b)) \leqslant \frac{a-2}{a-1}$ which implies that all sequences in $\Omega_{\nu}$ are admissible. ${ }^{9}$

Moreover, we have $\eta(a, b, \nu)>\eta(a, b, \nu(a, b)+0)>\frac{a-2}{a-1}$ when $\nu>\nu(a, b)$. Together with the fact that the supremum on $\Omega_{\nu}^{0}$ is attained, this implies that $\nu(a, b)$ is optimal.

Furthermore for any sequence in $\Omega_{\nu}^{0}$, we have

$$
\Psi_{e}(\theta)=-1+\sum_{k=1}^{\infty} a^{-k} \theta_{1}^{k}+\sum_{k=1}^{\infty} b^{-k}\left(1-\theta_{1}^{k}\right)
$$

Since the supremum on $\Omega_{\nu}^{0}$ is attained, this implies - using also relation (6) - that both maps $b \mapsto \eta(a, b, \nu)$ and $a \mapsto \eta(a, b, \nu)$ are strictly decreasing and continuous. By Lemma A.3, it follows that both maps $b \mapsto \nu(a, b)$ and $b \mapsto \nu(a, b)$ are increasing Devil's staircases. ${ }^{10}$

It remains to prove the ranges. We first show that $\nu(a, b)=0$ when $b$ is sufficiently close to 1 or when $a$ is sufficiently close to 2 . By definition, we have $\eta(a, b, \nu) \geqslant \phi_{\nu}(0)$ for every $\nu$. Using also that the function $\nu \mapsto \phi_{\nu}(0)$ is increasing and the property $\lim _{\nu \rightarrow 0^{+}}\lceil k \nu\rceil=1$ for every $k \geqslant 1$, it results that for all $\nu>0$ we have

$$
\eta(a, b, \nu)>\lim _{\nu \rightarrow 0^{+}} \phi_{\nu}(0)=\sum_{k=1}^{+\infty}\left(\frac{b-1}{b^{k+1}}-\frac{a-1}{a^{k+1}}\right)=\frac{1}{b}-\frac{1}{a}
$$

\footnotetext{
${ }^{9}$ If $\nu(a, b)=\frac{p}{q}$ is rational and the parameters are such that $\eta(\nu(a, b))<\frac{a-2}{a-1}$ then the set $\Omega_{\nu(a, b)}$ is also admissible. ${ }^{10}$ The proof is similar to the proof in [CF97a] that the fronts velocity is a Devil's staircase.
} 
Consequently, the condition $\frac{1}{b}-\frac{1}{a} \geqslant \frac{a-2}{a-1}$ implies that $\eta(a, b, \nu)>\frac{a-2}{a-1}$ for all $\nu>0$ and thus $\nu(a, b)=0$. It is immediate to check that this condition is satisfied when $b$ is sufficiently close to $1(a>2$ fixed), or when $a$ is sufficiently close to 2 (since we assume $b<2$ ).

Now we study the limits $b \rightarrow 2$ and $a \rightarrow+\infty$. Similar arguments to as before show that

$$
\eta(a, b, 1)=\phi_{1}(0)=\sum_{k=1}^{+\infty}\left(\frac{b-1}{b^{k+1}}-\frac{a-1}{a^{k+1}}\right) k=\frac{1}{b-1}-\frac{1}{a-1}
$$

By strict monotonicity of $\nu \mapsto \eta(a, b, \nu)$, this implies that $\nu(a, b)<1$ for every $a>2$ and $1<b<2$.

Let $a>2$ be fixed. By using that $k^{*}=1$ when $b$ is close to 2 and uniform convergence of the series defining $\phi_{\nu}(0)$, one shows that for every $\epsilon>0$ there exists $\delta(\epsilon)>0$ such that if $2-\delta(\epsilon)<b<2$, we have

$$
|\eta(a, b, \nu)-\eta(a, 2, \nu)|<\epsilon \quad \forall \nu \in[0,1]
$$

By strict monotonicity, we have $\eta(a, 2,1)-\eta(a, 2,1-\epsilon)>0$ for every $\epsilon>0$. It results that for $b>$ $2-\delta(\eta(a, 2,1)-\eta(a, 2,1-\epsilon))$ we have

$$
\eta(a, b, 1-\epsilon)<\eta(a, 2,1-\epsilon)+(\eta(a, 2,1)-\eta(a, 2,1-\epsilon))=\frac{a-2}{a-1}
$$

ie. $\nu(a, b) \geqslant 1-\epsilon$. This shows that $\lim _{b \rightarrow 2^{-}} \nu(a, b)=1$.

Let now $1<b<2$ be fixed. Explicit calculations show that we have

$$
\lim _{a \rightarrow+\infty}\left(\eta(a, b, 1-\epsilon)-\frac{a-2}{a-1}\right) \geqslant \frac{2-b}{b-1}-\sum_{k=1}^{+\infty} \frac{b-1}{b^{k+1}}\lfloor k \epsilon\rfloor
$$

and, by right continuity of $\lfloor\cdot\rfloor$, the latter is positive provided that $\epsilon$ is sufficiently small. Since the map $\nu \mapsto \eta(a, b, \nu)$ is strictly increasing we obtain that $\nu(+\infty, b):=\lim _{a \rightarrow+\infty} \nu(a, b)<1$ for every $1<b<2$.

Proof of Lemma A.3: Since $\Omega_{\nu_{1}} \subset \Omega_{\nu_{2}}$ when $\nu_{1}<\nu_{2}$, the map $\nu \mapsto \eta(\nu)$ is an increasing function. If $\nu \in[0,1] \backslash \mathbb{Q}$ then we have

$$
\lim _{\nu^{\prime} \rightarrow \nu}\left(\left\lceil(k-m) \nu^{\prime}\right\rceil+\left\lfloor m \nu^{\prime}\right\rfloor\right)=\lceil(k-m) \nu\rceil+\lfloor m \nu\rfloor, \quad m, k \geqslant 1
$$

which implies $\lim _{\nu^{\prime} \rightarrow \nu} \phi_{\nu^{\prime}}\left(m \nu^{\prime}-\left\lfloor m \nu^{\prime}\right\rfloor\right)=\phi_{\nu}(m \nu-\lfloor m \nu\rfloor)$ for every $m \geqslant 1$. Hence, $\eta(\cdot)$ is continuous at every irrational number.

We prove below the following inequality. For every $p<q$ coprime integers and every $m \in\{0, \cdots, q-1\}$ we have

$$
\phi_{\frac{p}{q}}\left(m \frac{p}{q}-\left\lfloor m \frac{p}{q}\right\rfloor\right)<\lim _{\nu \rightarrow \frac{p}{q}+} \phi_{\nu}(m \nu-\lfloor m \nu\rfloor)
$$

Obviously, this implies discontinuity of $\eta(\cdot)$ at every rational number. Actually, it also implies that this map is strictly increasing. Indeed given $\nu_{1}<\nu_{2}$, by density let $q$ be the smallest integer such that $\nu_{1} \leqslant \frac{p}{q}<\nu_{2}$ for some integer $p$ (which is relatively prime to $q$ ). Let $m \in\{0, \cdots, q-1\}$ be such that $\eta\left(\frac{p}{q}\right)=\phi \frac{p}{q}\left(m \frac{p}{q}-\left\lfloor m \frac{p}{q}\right\rfloor\right)$. By relation (8) and by definition of $\eta$, it follows that

$$
\eta\left(\frac{p}{q}\right)=\phi_{\frac{p}{q}}\left(m \frac{p}{q}-\left\lfloor m \frac{p}{q}\right\rfloor\right)<\lim _{\nu \rightarrow \frac{p}{q}+} \phi_{\nu}(m \nu-\lfloor m \nu\rfloor) \leqslant \lim _{\nu \rightarrow \frac{p}{q}+} \eta(\nu)
$$

and then $\eta\left(\nu_{1}\right) \leqslant \eta\left(\frac{p}{q}\right)<\eta\left(\nu_{2}\right)$.

It remains to show the inequality (8). By properties of left and right limits of the functions $\lceil\cdot\rceil$ and $\lfloor\cdot\rfloor$ we obtain

$$
\begin{aligned}
\lim _{\nu \rightarrow \frac{p}{q}+} \phi_{\nu}(m \nu-\lfloor m \nu\rfloor)-\phi_{\frac{p}{q}}\left(m \frac{p}{q}-\left\lfloor m \frac{p}{q}\right\rfloor\right) & =\lim _{\nu \rightarrow \frac{p}{q}+} \sum_{k=1}^{+\infty}\left(\frac{b-1}{b^{k+1}}-\frac{a-1}{a^{k+1}}\right)\left(\lceil(k-m) \nu\rceil-\left\lceil(k-m) \frac{p}{q}\right\rceil\right) \\
& =\sum_{k=1}^{+\infty}\left(\frac{b-1}{b^{q k+m+1}}-\frac{a-1}{a^{q k+m+1}}\right) \\
& =\frac{1}{\sum_{k=0}^{q-1} b^{m+k+1}}-\frac{1}{\sum_{k=0}^{q-1} a^{m+k+1}}>0
\end{aligned}
$$


where the last inequality relies on the fact that $b<a$.

\section{B Proof of Proposition 4.2}

The properties listed in Proposition 4.2 are direct consequence of equation (14). This equation follows from an induction relation (12) on the number $N_{t}$ of words of length $t$ in $\Omega_{\nu}$. In order to obtain this relation, we decompose words of length $t$ according to their prefixes.

Since the entropy of $\left(\Omega_{0}, \sigma\right)$ can be computed directly, we may assume that $\nu>0$ throughout the proof. Given $k \geqslant 0$, consider a word $\omega_{0} \cdots \omega_{k-1} \in\{-1,0,1\}^{k}$ such that

$$
\left|\sum_{t=t_{1}}^{t_{2}-1} \omega_{t}\right| \leqslant\left\lceil\left(t_{2}-t_{1}\right) \nu\right\rceil
$$

for all $0 \leqslant t_{1}<t_{2} \leqslant k$ (ie. $t_{2}$ is bounded above by the word length). Given $t \geqslant k$ let $N_{t}^{\omega_{0} \cdots \omega_{k-1}}$ be the number of words of length $t$ in $\Omega_{\nu}$ such that

$$
\theta_{0}^{i}-\theta_{1}^{i}=\omega_{i} \quad \forall 0 \leqslant i \leqslant k-1
$$

In particular, we shall consider $N_{t}^{\varkappa_{0} \cdots \varkappa_{k-1}}$ where $\varkappa_{k}=\lceil(k+1) \nu\rceil-\lceil k \nu\rceil$. For the sake of convenience, we shall also set $N_{0}=1, N_{t}=0$ if $t<0$ and $N_{t}^{\omega_{0} \cdots \omega_{k-1}}=0$ if $t<k$ or if $\omega_{0} \cdots \omega_{k-1}$ does not satisfies the condition (9).

The quantity $N_{t}^{\varkappa_{0} \cdots \varkappa_{k-1}}$ has the following properties.

Lemma B.1. (1) $N_{t}^{\varkappa_{0} \cdots \varkappa_{k-1}(-1)}=2^{k-\lceil k \nu\rceil} N_{t-k}^{-1}$ for all $t \in \mathbb{Z}$ and $k \geqslant 0$.

(2) $N_{t}^{\varkappa_{0} \cdots \varkappa_{k-1} 0}=2^{k-\lceil k \nu\rceil} N_{t-k}^{0}$ for all $t \in \mathbb{Z}$ and $k \geqslant 0$ such that $\varkappa_{k}=1$.

(3) $N_{t}^{\varkappa_{0} \cdots \varkappa_{k-1} 1}=0$ for all $t \in \mathbb{Z}$ and $k \geqslant 0$ such that $\varkappa_{k}=0$.

(4) If $\nu=\frac{p}{q}$, then $N_{t}^{\varkappa_{0} \cdots \varkappa_{q-1}}=2^{q-p} N_{t-q}$ for all $t \in \mathbb{Z}$.

Proof: (1) One checks that for any $\omega_{0}^{\prime} \cdots \omega_{i-1}^{\prime} \in\{-1,0,1\}^{i}$ with $\omega_{0}^{\prime}=-1$ and satisfying the condition (9), the concatenation

$$
\omega_{0} \cdots \omega_{k+i-1}:=\varkappa_{0} \cdots \varkappa_{k-1} \omega_{0}^{\prime} \cdots \omega_{i-1}^{\prime}
$$

also satisfies the condition (9). In particular, for $t_{1}<k \leqslant t_{2}-2$ we have

$$
\begin{aligned}
&\left|\sum_{t=t_{1}}^{t_{2}-1} \omega_{t}\right|=\left|\sum_{t=t_{1}}^{k-1} \varkappa_{t}-1+\sum_{t=k+1}^{t_{2}-1} \omega_{t-(k+1)}^{\prime}\right| \\
& \leqslant\lceil k \nu\rceil-\left\lceil t_{1} \nu\right\rceil-1+\left\lceil\left(t_{2}-k-1\right) \nu\right\rceil \leqslant\left\lceil\left(t_{2}-t_{1}\right) \nu\right\rceil
\end{aligned}
$$

This implies that $N_{t}^{\varkappa_{0} \cdots \varkappa_{k-1}(-1)}=N_{k}^{\varkappa_{0} \cdots \varkappa_{k-1}} N_{t-k}^{-1}$.

On the other hand, the word $\varkappa_{0} \cdots \varkappa_{k-1}$ contains $\lceil k \nu\rceil$ symbols 1 and $k-\lceil k \nu\rceil$ symbols 0 . Hence $N_{k}^{\varkappa_{0} \cdots \varkappa_{k-1}}=$ $2^{k-\lceil k \nu\rceil}$ and property (1) follows.

(2) The proof is similar to that of (1). One checks that for any $\omega_{0}^{\prime} \cdots \omega_{i-1}^{\prime} \in\{-1,0,1\}^{i}$ with $\omega_{0}^{\prime}=0$ and satisfying the condition (9), the concatenation $\varkappa_{0} \cdots \varkappa_{k-1} \omega_{0}^{\prime} \cdots \omega_{i-1}^{\prime}$ also satisfies the condition (9) provided that $\varkappa_{k}=1$.

(3) We have $\sum_{t=0}^{k-1} \varkappa_{t}=\lceil k \nu\rceil$. Therefore, we have $\sum_{t=0}^{k-1} \varkappa_{t}+1>\lceil(k+1) \nu\rceil$ and then $N_{t}^{\varkappa_{0} \cdots \varkappa_{k-1} 1}=0$ if $\varkappa_{k}=0$.

(4) As before it suffices to show that $\varkappa_{0} \cdots \varkappa_{q-1} \omega_{0}^{\prime} \cdots \omega_{i-1}^{\prime}$ satisfies the condition (9) whenever $\omega_{0}^{\prime} \cdots \omega_{i-1}^{\prime}$ does satisfy the same condition.

Similarly, one proves that

$$
N_{t}^{0}=2 N_{t-1}, N_{t}^{-1}=N_{t}^{1}, \text { and } N_{t}=N_{t}^{-1}+N_{t}^{0}+N_{t}^{1} \text { if } t \geqslant 1
$$


and

$$
N_{t}^{\varkappa_{0} \cdots \varkappa_{k-1}}=N_{t}^{\varkappa_{0} \cdots \varkappa_{k-1}(-1)}+N_{t}^{\varkappa_{0} \cdots \varkappa_{k-1} 0}+N_{t}^{\varkappa_{0} \cdots \varkappa_{k-1} 1} \forall t>k>0
$$

If $\varkappa_{k}=1$ then by Lemma B.1 (2) and relation (10) we obtain

$$
N_{t}^{\varkappa_{0} \cdots \varkappa_{k-1} 0}+N_{t}^{\varkappa_{0} \cdots \varkappa_{k-1} 1}=2^{k-\lceil k \nu\rceil+1} N_{t-k-1}+N_{t}^{\varkappa_{0} \cdots \varkappa_{k}}
$$

Otherwise we have $\varkappa_{k}=0$ and then $N_{t}^{\varkappa_{0} \cdots \varkappa_{k-1} 0}=N_{t}^{\varkappa_{0} \cdots \varkappa_{k}}$ and $N_{t}^{\varkappa_{0} \cdots \varkappa_{k-1} 1}=0$ by Lemma B.1 (3). Using also Lemma B.1 (1) and relation (10) once again, we obtain the following condensed induction relation

$$
N_{t}^{\varkappa_{0} \cdots \varkappa_{k-1}}=2^{k-\lceil k \nu\rceil} N_{t-k}^{1}+2^{k-\lceil k \nu\rceil+1} \varkappa_{k} N_{t-k-1}+N_{t}^{\varkappa_{0} \cdots \varkappa_{k}}
$$

This relation extends to $k=0$ if we define $N_{t}^{\varkappa_{0} \cdots \varkappa_{k-1}}=N_{t}$ in this case.

By summing relation (11) for $k$ from 0 to $n-1$ and by using that $N_{t}^{1}=\frac{N_{t}}{2}-N_{t-1}$ and $2^{-\varkappa_{k}}+2 \varkappa_{k}-1=\frac{3}{2} \varkappa_{k}$, we obtain the desired induction relation ${ }^{11}$

$$
N_{t}=2 N_{t}^{\varkappa_{0} \cdots \varkappa_{n-1}}-2^{n-\lceil n \nu\rceil} N_{t-n}+3 \sum_{k=0}^{n-1} 2^{k-\lceil k \nu\rceil} \varkappa_{k} N_{t-k-1} \forall t \geqslant n
$$

In order to deduce (14) from (12) we start by considering the case where $\nu=\frac{p}{q}$ is rational and we set $n=q$. By Lemma B.1 (4) we obtain

$$
N_{t}=2^{q-p} N_{t-q}+3 \sum_{k=0}^{q-1} 2^{k-\lceil k \nu\rceil} \varkappa_{k} N_{t-k-1}
$$

This relation can be written as $\mathbf{N}_{t}=A_{p, q} \mathbf{N}_{t-1}$ where $\mathbf{N}_{t}=\left(N_{t}, \cdots, N_{t-q+1}\right)$ is a $q$-dimensional integer vector and $A_{p, q}$ is a companion matrix (and hence primitive) with non negative entries. By the Perron-Frobenius theorem, it has a unique eigenvalue, say $\frac{1}{x_{\frac{p}{q}}}$, which is equal to the spectral radius. The corresponding eigenvector writes $\left(C x_{\frac{p}{q}}^{-q+1}, \cdots, C\right)$ for some constant $C>0$. As a consequence we have

$$
h\left(\Omega_{\frac{p}{q}}, \sigma\right)=-\log x_{\frac{p}{q}} \quad \text { and } \quad \lim _{t \rightarrow \infty} \frac{N_{t-k}}{N_{t}}=x_{\frac{p}{q}}^{k} \forall k \geqslant 1
$$

In addition, equation (10) implies $N_{t} \geqslant 2^{t}$. Together with $\nu>0$, it results that $\lim _{t \rightarrow \infty} \frac{2^{t-\lceil t \nu\rceil}}{N_{t}}=0$.

Dividing by $N_{t}$ in equation (12) with $n=t$, we conclude that when $\nu=\frac{p}{q}$, the quantity $x_{\nu}$ is the positive root of the equation

$$
1=3 \sum_{k=0}^{+\infty} 2^{k-\lceil k \nu\rceil} \varkappa_{k} x^{k+1}
$$

Now the map $\nu \mapsto x_{\nu}$ is continuous at every irrational number. Moreover by monotonicity of the entropy, we have

$$
-\log x \frac{p_{1}}{q_{1}} \leqslant h\left(\Omega_{\nu}, \sigma\right) \leqslant-\log x \frac{p_{2}}{q_{2}}
$$

for any $\nu \in \mathbb{R} \backslash \mathbb{Q}$ and $\frac{p_{1}}{q_{1}}<\nu<\frac{p_{2}}{q_{2}}$. Continuity then implies

$$
-\lim _{\frac{p_{1} \rightarrow \nu^{-}}{q_{1}}} \log x_{\frac{p_{1}}{q_{1}}}=-\log x_{\nu} \leqslant h\left(\Omega_{\nu}, \sigma\right) \leqslant-\log x_{\nu}=-\lim _{\frac{p_{2}}{q_{2}} \rightarrow \nu^{+}} \log x \frac{p_{2}}{q_{2}}
$$

which shows that the relation $h\left(\Omega_{\nu}, \sigma\right)=-\log x_{\nu}$ also holds when $\nu$ is irrational.

Since for $\nu>0$, those $k$ for which $\varkappa_{k}=1$ take the form $\left\lfloor\frac{j}{\nu}\right\rfloor$, equation (13) can be written as follows

$$
1=3 x \sum_{j=0}^{+\infty} \frac{(2 x)^{\left\lfloor\frac{j}{\nu}\right\rfloor}}{2^{j}}
$$

\footnotetext{
${ }^{11}$ Since we have $N_{t}^{\varkappa_{0} \cdots \varkappa_{t-1}}=2^{t-\lceil t \nu\rceil}$, this relation indeed becomes an induction relation on $N_{t}$ for $n=t$.
} 
which is the desired equation for $x_{\nu}=e^{-h\left(\Omega_{\nu}, \sigma\right)}$.

Strict monotonicity and continuity properties of $\nu \mapsto h\left(\Omega_{\nu}, \sigma\right)$ then easily follow. (The proof of left continuity uses uniform continuity of the mapping $x \mapsto 3 x \sum_{j=0}^{+\infty} \frac{\left(2 x x^{\left\lfloor\frac{j}{\nu}\right\rfloor}\right.}{2^{j}}$.) Moreover $\Omega_{0}=\{00,11\}^{\mathbb{N}}$ which implies $h\left(\Omega_{0}, \sigma\right)=\log 2$. Similarly, we have $\Omega_{1}=\{01,00,11,10\}^{\mathbb{N}}$ which implies $h\left(\Omega_{1}, \sigma\right)=\log 4$. Finally we notice that

$$
\begin{aligned}
3 x \sum_{j=0}^{+\infty} \frac{(2 x)^{\left\lfloor\frac{j}{\nu}\right\rfloor}}{2^{j}} & <\frac{3}{4} \sum_{j=0}^{+\infty} \frac{1}{4^{j}}=1 \text { if } x=\frac{1}{4} \\
& =3 x+3 x \sum_{j=1}^{+\infty} \frac{(2 x)^{\left\lfloor\frac{j}{\nu}\right\rfloor}}{2^{j}}>1 \text { if } x=\frac{1}{3}
\end{aligned}
$$

which implies $\log 3<h\left(\Omega_{\nu}, \sigma\right)<\log 4$ for all $\nu \in(0,1)$. The proposition is proved.

\section{Equation for $h\left(\Xi_{n}, \sigma\right)$}

As in the proof of Proposition 4.2, an induction relation on the number $N_{t}$ of words of length $t$ in $\Xi_{n}$ is obtained by decomposing the words according to their prefixes. Since the words of length $n$ in this set are identical to those in $\Omega_{\frac{n}{n+1}}$, the relation (11) for $0 \leqslant k \leqslant n-1$ extends to the present case, ie. we have ${ }^{12}$

$$
N_{t}^{\varkappa_{0} \cdots \varkappa_{k-1}}=N_{t-k}^{1}+2 N_{t-k-1}+N_{t}^{\varkappa_{0} \cdots \varkappa_{k}}
$$

However for $k=n$ the right hand side contains an additional term because $N_{t}^{\varkappa_{0} \cdots \varkappa_{n-1} 1} \neq 0($ when $t \geqslant n+1)$, ie. we have

$$
N_{t}^{\varkappa_{0} \cdots \varkappa_{n-1}}=N_{t-n}^{1}+2 N_{t-n-1}+N_{t}^{\varkappa_{0} \cdots \varkappa_{n}}+N_{t}^{\varkappa_{0} \cdots \varkappa_{n-1} 1}
$$

Then a similar computation to the one in Appendix B leading to relation (12) leads to

$$
N_{t}=3 \sum_{k=1}^{n} N_{t-k}+2 N_{t-n-1}+2 N_{t}^{\varkappa_{0} \cdots \varkappa_{n-1} 1}
$$

Now by definition of $\Xi_{n}$ we have

$$
N_{t}^{\varkappa_{0} \cdots \varkappa_{n-1} 1}=\sum_{k=0}^{n-1}\left(N_{t-n-1}^{(-1) \varkappa_{1} \cdots \varkappa_{k}(-1)}+N_{t-n-1}^{(-1) \varkappa_{1} \cdots \varkappa_{k} 0}\right)
$$

As in Appendix B, a reasoning based on Lemma B.1 then implies that

$$
N_{t}^{\varkappa_{0} \cdots \varkappa_{n-1} 1}=\frac{1}{2} N_{t-n-2}+\frac{3}{2} \sum_{k=n+3}^{2 n+1} N_{t-k}+N_{t-2 n-2}
$$

Once again as before, a reasoning based on Perron-Frobenius theorem shows that $h\left(\Xi_{n}, \sigma\right)=-\log x_{n}^{+}$where $x_{n}^{+}$is the positive root of the following equation

$$
1=3 \sum_{k=1}^{n} x^{k}+2 x^{n+1}+x^{n+2}+3 \sum_{k=n+3}^{2 n+1} x^{k}+2 x^{2 n+2}
$$

\footnotetext{
${ }^{12}$ In this computation the notation $N_{t}^{\varkappa_{0} \cdots \varkappa_{k-1}}$ is somehow redundant because $\varkappa_{k}=1$ for all $0 \leqslant k \leqslant n-1$. The reason to employ it is that is it particularly adapted to the computation of the entropy of extensions of $\Xi_{n}$.
} 


\section{Proofs of results in section 4.2.2}

\section{D.1 Proof of Theorem 4.5}

The proof is similar (and simpler) to that of Theorem 4.1. We have to compute the quantity $\sup _{\theta \in \Lambda_{\nu}^{0}} \Psi_{e}(\theta)$ where $\Lambda_{\nu}^{0} \in \Lambda_{\nu}$ is the subset of sequences such that $\theta_{0}^{0}=0$. Considering that a sequence in $\Lambda_{\nu}^{0}$ is composed of two individual sequences, $\theta=\left(\left\{\theta_{0}^{t}\right\}_{t \in \mathbb{N}},\left\{\theta_{1}^{t}\right\}_{t \in \mathbb{N}}\right)$, the expression of $\Psi_{e}$ can be split into the sum of two terms, $\Psi_{e}(\theta)=\Psi_{e}^{0}\left(\left\{\theta_{0}^{t}\right\}_{t \in \mathbb{N}}\right)+\Psi_{e}^{1}\left(\left\{\theta_{1}^{t}\right\}_{t \in \mathbb{N}}\right)$ where

$$
\Psi_{e}^{0}(\vartheta)=\sum_{k=0}^{\infty}\left(a^{-k}+b^{-k}\right) \vartheta^{k} \quad \text { and } \quad \Psi_{e}^{1}(\vartheta)=-\frac{a}{a-1}+\sum_{k=0}^{\infty}\left(a^{-k}-b^{-k}\right) \vartheta^{k}
$$

The product structure $\Lambda_{\nu}=\lambda_{\nu} \times \lambda_{\nu}$ then implies the following decomposition

$$
\sup _{\theta \in \Lambda_{\nu}^{0}} \Psi_{e}(\theta)=\sup _{\vartheta \in \lambda_{\nu}^{0}} \Psi_{e}^{0}(\vartheta)+\sup _{\vartheta \in \lambda_{\nu}} \Psi_{e}^{1}(\vartheta)
$$

where $\lambda_{\nu}^{0} \subset \lambda_{\nu}$ is the subset of sequences starting with 0 . Moreover the inequality $b<a$ implies that

$$
\sup _{\vartheta \in \lambda_{\nu}} \Psi_{e}^{1}(\vartheta)=-\frac{a}{a-1}
$$

The computation of $\sup _{\vartheta \in \lambda_{\nu}^{0}} \Psi_{e}^{0}(\vartheta)$ follows the same lines as in Appendix A. By definition of $\lambda_{\nu}$, we have $\sup _{\vartheta \in \lambda_{\nu}^{0}} \Psi_{e}^{0}(\vartheta)=\sup _{J \in \Theta_{\nu}} \Phi_{\Lambda}(J)$ where $\Phi_{\Lambda}(J)=\sum_{k=1}^{+\infty}\left(\frac{b-1}{b^{k+1}}+\frac{a-1}{a^{k+1}}\right) J_{k}$ and again

$$
\Theta_{\nu}=\left\{\left\{J_{k}\right\}_{k \in \mathbb{N}} \in \mathbb{N}^{\mathbb{N}}: J_{0}=0, J_{k} \leqslant J_{k+1}, J_{k_{2}}-J_{k_{1}} \leqslant\left\lceil\left(k_{2}-k_{1}\right) \nu\right\rceil \forall 0 \leqslant k_{1}<k_{2}\right\}
$$

Since $\frac{b-1}{b^{k+1}}+\frac{a-1}{a^{k+1}}>0$, it is immediate to show that

$$
\sup _{J \in \Theta_{\nu}} \Phi_{\Lambda}(J)=\eta_{\Lambda}(\nu):=\sum_{k=1}^{+\infty}\left(\frac{b-1}{b^{k+1}}+\frac{a-1}{a^{k+1}}\right)\lceil k \nu\rceil
$$

Similarly as in Lemma A.3, one can show that the map $\nu \mapsto \eta_{\Lambda}(\nu)$ is strictly increasing, continuous at every irrational number and discontinuous at every rational number. We conclude that desired (optimal) quantity $\nu_{\Lambda}(a, b)$ is defined by the relation

$$
\nu_{\Lambda}(a, b)=\sup \left\{\nu \in\left[\frac{1}{2}, 1\right]: \eta_{\Lambda}(a, b, \nu) \leqslant \frac{a}{a-1}\right\}
$$

where the explicit dependence on $a$ and $b$ has been included in the quantity $\eta_{\Lambda}(\cdot)$. Furthermore, the expression of $\Psi_{e}^{0}$ implies that both function $b \mapsto \eta_{\Lambda}(a, b, \nu)$ and $a \mapsto \eta_{\Lambda}(a, b, \nu)$ are strictly decreasing and continuous. Consequently, both maps $b \mapsto \nu_{\Lambda}(a, b)$ and $a \mapsto \nu_{\Lambda}(a, b)$ are increasing Devil's staircases.

As in the proof of Theorem 4.1, we compute the ranges. Using that $\lim _{\nu \rightarrow \frac{1}{2}^{+}}\lceil k \nu\rceil=\left\lceil\frac{k+1}{2}\right\rceil$ for every $k \geqslant 1$, explicit calculations lead to the following result

$$
\lim _{\nu \rightarrow \frac{1}{2}^{+}} \eta_{\Lambda}(a, b, \nu)=\frac{b^{2}+b-1}{b\left(b^{2}-1\right)}+\frac{a^{2}+a-1}{a\left(a^{2}-1\right)}
$$

This quantity diverges when $b \rightarrow 1$ and is smaller than $\frac{a}{a-1}$ when $b$ is close to 2 . Moreover it remains bounded when $a$ changes in $(2,+\infty)$. Together with the relation (15) and monotonicity of $b \mapsto \nu_{\Lambda}(a, b)$ it results that, for every $a>2$ there exists $1<b_{0}(a)<2$ such that $\nu_{\Lambda}(a, b)=\frac{1}{2}$ whenever $1<b \leqslant b_{0}(a)$. The monotonicity of $a \mapsto \nu_{\Lambda}(a, b)$ implies that the map $a \mapsto b_{0}(a)$ is decreasing and we have $b^{*}:=\lim _{a \rightarrow 2^{+}} b_{0}(a)>1$. (The number $b^{*} \simeq 1.66$ is the (unique positive) root of some third order polynomial.)

Consequently, if $b \geqslant b^{*}$ then $\lim _{a \rightarrow 2^{+}} \nu_{\Lambda}(a, b)>\frac{1}{2}$ and if $1<b<b^{*}$ then $\nu_{\Lambda}(a, b)=\frac{1}{2}$ provided that $2<a \leqslant$ $b_{0}^{-1}(b)$. Notice that $b_{0}^{-1}(b)=+\infty$ if $b$ is close to 1 .

Furthermore, based on the computation of $\eta_{\Lambda}(a, b, 1)$, similar arguments to as in the proof of Theorem 4.1 show that $\lim _{b \rightarrow 2^{-}} \nu_{\Lambda}(a, b)=1$ and $\lim _{a \rightarrow+\infty} \nu_{\Lambda}(a, b)<1$. 


\section{D.2 Proof of Proposition 4.6}

The proof follow the same techniques as in Appendices B and C. We compute an induction relation for the number $n_{t}$ of words of length $t$ in $\lambda_{\nu}$ by decomposing the words according to their prefixes.

Using similar notation to as before, we have

$$
n_{t}^{\varkappa_{0} \cdots \varkappa_{k-1}}=n_{t}^{\varkappa_{0} \cdots \varkappa_{k-1} 0}+n_{t}^{\varkappa_{0} \cdots \varkappa_{k-1} 1}
$$

Now when $\varkappa_{k}=1$, given any word $\omega_{0}^{\prime} \cdots \omega_{i-1}^{\prime} \in\{0,1\}^{i}$ in $\lambda_{\nu}$ such that $\omega_{0}^{\prime}=0$, the concatenation $\varkappa_{0} \cdots \varkappa_{k-1} \omega_{0}^{\prime} \cdots \omega_{i-1}^{\prime}$ also belong to $\lambda_{\nu}$. This implies that $n_{t}^{\varkappa_{0} \cdots \varkappa_{k-1} 0}=n_{k}^{\varkappa_{0} \cdots \varkappa_{k-1}} n_{t-k}^{0}$.

Moreover it is direct to show that $n_{k}^{\varkappa_{0} \cdots \varkappa_{k-1}}=1$ for all $k \geqslant 0$ and that

$$
n_{t}=n_{t}^{0}+n_{t}^{1}=2 n_{t}^{0}
$$

Collecting these results we obtain

$$
n_{t}^{\varkappa_{0} \cdots \varkappa_{k-1}}=\frac{\varkappa_{k}}{2} n_{t-k}+n_{t}^{\varkappa_{0} \cdots \varkappa_{k}}
$$

Summing for $k$ from 0 to $t-1$ yields

$$
n_{t}=1+\frac{1}{2} \sum_{k=0}^{t-1} \varkappa_{k} n_{t-k}
$$

Reasoning similarly as in Appendix B, we obtain that the entropy of $\lambda_{\nu}$ is the logarithm of the positive root of the equation

$$
2=\sum_{j=0}^{+\infty} x^{\left\lfloor\frac{j}{\nu}\right\rfloor}
$$

Strict monotonicity and continuity properties then easily follow. Moreover one checks that for $\nu=\frac{1}{2}$ the positive root of this equation is $\frac{1}{\sqrt{2}}$ which implies that $h\left(\Lambda_{\frac{1}{2}}, \sigma\right)=\log 2$. In addition, we have $\Lambda_{1} \stackrel{\frac{1}{2}}{=}$ $\{01,00,11,10\}^{\mathbb{N}}$.

\section{D.3 Proof of Corollary 4.7}

In proving the ranges of the functions involved in Theorem 4.1, we proved the existence $b_{1}(a)$, with $\lim _{a \rightarrow 2^{+}} b_{1}(a)=2$, such that $\nu(a, b)=0$ when $1<b \leqslant b_{1}(a)$. From the proof of Theorem 4.5 , we have $\lim _{a \rightarrow 2^{+}} b_{0}(a)<2$. It results that when $a$ is sufficiently close to 2 , we have $b_{0}(a)<b_{1}(a)$ and then

$$
h\left(\Lambda_{\nu_{\Lambda}(a, b)}, \sigma\right)>h\left(\Omega_{\left.\nu_{(} a, b\right)}, \sigma\right)=\log 2 \quad \text { when } b_{0}(a)<b \leqslant b_{1}(a)
$$

and using that $h\left(\Lambda_{\frac{1}{2}}, \sigma\right)=\log 2$,

$$
h\left(\Lambda_{\nu_{\Lambda}(a, b)}, \sigma\right)=h\left(\Omega_{\left.\nu_{(} a, b\right)}, \sigma\right)=\log 2 \quad \text { when } b \leqslant b_{0}(a)
$$

The rest of the proof is standard.

\section{E $\quad$ Proof of Proposition 5.3}

Every sequence in $\Gamma_{n}$ is admissible iff $\sup _{\theta \in \Gamma_{n}: \theta_{0}^{0}=0} \Psi_{h}(\theta)<0$. In order to compute this supremum we shall consider separately the cases $\theta_{0}^{0}=00$ and $\theta_{0}^{0}=01$ and we shall consider separately the quantities $S(\cdot)$ and $D_{h}(\cdot)$.

As a preliminary remark, notice that by definition of $\Psi_{h}$ this supremum is attained in the subset $\Gamma_{n}^{\prime}$ of sequences for which every homogeneous symbol is equal to 11 , excepted maybe $\theta^{0}$. Indeed replacing 00 by 11 changes a sequence in $\Gamma_{n}$ to another sequence in this set and increases $\Psi_{h}$. 
Consider the lexicographic ordering in $\{01,11,10\}^{\mathbb{N}^{*}}$ with basic ordering $01=10<11$. Since $a>2$, it follows that the mapping $\left\{\theta^{k}\right\}_{k=1}^{\infty} \mapsto \sum_{k=1}^{\infty} a^{-k}\left(\theta_{0}^{k}+\theta_{1}^{k}-1\right)$ is increasing. Introducing the mapping $\left\{\theta^{k}\right\}_{k=0}^{\infty} \mapsto$ $S^{+}\left(\left\{\theta^{k}\right\}_{k=0}^{\infty}\right):=\sum_{k=0}^{\infty} a^{-k}\left(\theta_{0}^{k}+\theta_{1}^{k}-1\right)$ it results that

$$
\sup _{\theta \in \Gamma_{n}^{\prime}: \theta^{0}=00} S(\theta)=S^{+}\left(0011^{n-1}\left(1011^{n} 0111^{n}\right)^{\infty}\right)=S^{+}\left(0011^{n-1}\left(0111^{n} 1011^{n}\right)^{\infty}\right)
$$

and

$$
\sup _{\theta \in \Gamma_{n}^{\prime}: \theta^{0}=01} S(\theta)=S^{+}\left(\left(0111^{n} 1011^{n}\right)^{\infty}\right)
$$

Consider now the mapping $\underline{\theta}:=\left\{\theta^{k}\right\}_{k=-\infty}^{0} \mapsto D_{h}^{-}(\underline{\theta}):=\sum_{k=1}^{\infty} b^{k}\left(\theta_{1}^{-k}-\theta_{0}^{-k}\right)$ (which is independent of $\theta^{0}$ ). We want to compute the supremum of $D_{h}^{-}$over $\Gamma_{n}^{00}$ and over $\Gamma_{n}^{01}$ where

$$
\Gamma_{n}^{00}=\left\{\left\{\theta^{k}\right\}_{k=-\infty}^{0}:\left\{\theta^{k}\right\}_{k \in \mathbb{Z}} \in \Gamma_{n}^{\prime} \text { and } \theta^{0}=00\right\}
$$

and

$$
\Gamma_{n}^{01}=\left\{\left\{\theta^{k}\right\}_{k=-\infty}^{0}:\left\{\theta^{k}\right\}_{k \in \mathbb{Z}} \in \Gamma_{n}^{\prime} \text { and } \theta^{0}=01\right\}
$$

We proceed similarly as in the beginning of the proof of Theorem 4.2. Define the quantity

$$
t_{01}(\underline{\theta})=\sup \left\{k \leqslant-1: \theta^{k} \theta^{k+1}=0111\right\}
$$

and the mapping $P$ on $\{01,00,11,10\}^{\mathbb{Z}^{-}}$as follows. If $t_{01}(\underline{\theta})=-\infty$ then $P(\underline{\theta})=\underline{\theta}$. Otherwise we set

$$
P(\underline{\theta})^{t}=\left\{\begin{array}{lll}
\underline{\theta}^{t} & \text { if } & t_{01}(\underline{\theta})+1<t \leqslant 0 \\
\underline{\theta}^{t-1} & \text { if } & t \leqslant t_{01}(\underline{\theta})+1
\end{array}\right.
$$

This map has the following properties

- $P\left(\Gamma_{n}^{00}\right) \subset \Gamma_{n}^{00}$ and $P\left(\Gamma_{n}^{01}\right) \subset \Gamma_{n}^{01}$.

- $D_{h}^{-}(\underline{\theta}) \leqslant D_{h}^{-} \circ P(\underline{\theta})$.

- The limit $\lim _{n \rightarrow \infty} P^{n}(\underline{\theta})=: P^{\infty}(\underline{\theta})$ exists and we have $t_{01} \circ P^{\infty}(\underline{\theta})=-\infty$ for every $\underline{\theta}$.

Similarly, define the quantity

$$
t_{10}(\underline{\theta})=\sup \left\{k \leqslant-1: \theta^{k}=10 \text { and } \exists i \leqslant n: \theta^{k+i}=01\right\}
$$

and the mapping $Q$ on $\{01,00,11,10\}^{\mathbb{Z}^{-}}$as follows. If $t_{10}(\underline{\theta})=-\infty$ then $Q(\underline{\theta})=\underline{\theta}$. Otherwise we set

$$
Q(\underline{\theta})^{t}=\left\{\begin{array}{cll}
\underline{\theta}^{t} & \text { if } & t_{10}(\underline{\theta})+1 \leqslant t \leqslant 0 \\
11 & \text { if } t=t_{10}(\underline{\theta}) \\
\underline{\theta}^{t+1} & \text { if } t \leqslant t_{10}(\underline{\theta})-1
\end{array}\right.
$$

which has similar properties to $P$. In particular, the relation $D_{h}^{-}(\underline{\theta}) \leqslant D_{h}^{-} \circ Q(\underline{\theta})$ follows from the fact that, for the sequences in $\Gamma_{n}$, the heterogeneous symbols alternate. As a consequence, the limit map $Q^{\infty}$ is such that $t_{01} \circ Q^{\infty}(\underline{\theta})=-\infty$ for every $\underline{\theta}$. Consequently, we obtain

$$
Q^{\infty} \circ P^{\infty}(\underline{\theta})={ }^{\infty}\left(1011^{n} 01\right)
$$

for any $\underline{\theta} \in \Gamma_{n}^{01}$ which yields

$$
\sup _{\underline{\theta} \in \Gamma_{n}^{01}} D_{h}^{-}(\underline{\theta})=D_{h}^{-}\left({ }^{\infty}\left(1011^{n} 01\right)\right)
$$

Moreover we have

$$
Q^{\infty} \circ P^{\infty}(\underline{\theta})={ }^{\infty}\left(1011^{n} 01\right) 00
$$


for any $\underline{\theta} \in \Gamma_{n}^{00}$ whose first heterogeneous symbol is 01 . Since $D_{h}^{-}(\underline{\theta})>0$ for any such sequence and since $D_{h}^{-}(\underline{\theta})<0$ for any $\underline{\theta} \in \Gamma_{n}^{00}$ whose first heterogeneous symbol is 10 , we conclude that

$$
\sup _{\underline{\theta} \in \Gamma_{n}^{00}} D_{h}^{-}(\underline{\theta})=D_{h}^{-}\left({ }^{\infty}\left(1011^{n} 01\right) 00\right)
$$

Now, the sequence ${ }^{\infty}\left(1011^{n} 01\right) 0011^{n-1}\left(1011^{n} 0111^{n}\right)^{\infty}$ belongs to $\Gamma_{n}^{\prime}$. Thus we have

$$
m_{1}(a, b, n):=\sup _{\theta \in \Gamma_{n}^{\prime}: \theta^{0}=00} \Psi_{h}(\theta)=D_{h}^{-}\left({ }^{\infty}\left(1011^{n} 01\right) 00\right)+S^{+}\left(0011^{n-1}\left(1011^{n} 0111^{n}\right)^{\infty}\right)
$$

Similarly, we conclude in the case $\theta^{0}=01$ that

$$
m_{2}(a, b, n):=\sup _{\theta \in \Gamma_{n}^{\prime}: \theta^{0}=01} \Psi_{h}(\theta)=D_{h}^{-}\left({ }^{\infty}\left(1011^{n} 01\right)\right)+S^{+}\left(\left(0111^{n} 1011^{n}\right)^{\infty}\right)
$$

Finally explicit calculations of these quantities show that, for every $a>2$ and $0<b<1$, we have $m_{1}(a, b, n)<$ $m_{2}(a, b, n)$ and

$$
m_{2}(a, b, n)=\frac{1}{a-1}-\frac{1}{a^{n+1}-1}-\frac{b^{n+1}(1-b)}{1-b^{n+2}}
$$

In other words $\sup _{\theta \in \Gamma_{n}: \theta_{0}^{0}=0} \Psi_{h}(\theta)=m_{2}(a, b, n)$ and then

$$
n^{\prime}(a, b)=\max \left\{n \in \mathbb{N}: m_{2}(a, b, n)<0\right\}
$$

This quantity is well-defined since $m_{2}(a, b, 0)$ is always negative and $\lim _{n \rightarrow+\infty} m_{2}(a, b, n)>0$.

The mapping $b \mapsto m_{2}(a, b, n)$ is decreasing on $(0,1)$, so the mapping $b \mapsto n^{\prime}(a, b)$ is increasing. Similarly, $a \mapsto m_{2}(a, b, n)$ is decreasing so $a \mapsto n^{\prime}(a, b)$ is increasing.

Moreover we have $m_{2}(a, 1-0,2)<0$ (and then $\left.n^{\prime}(a, 1-0) \geqslant 2\right)$ provided that $a^{3}-4 a^{2}-4 a-1>0$. Since $\lim _{n \rightarrow \infty} m_{2}(a, 1-0, n)>0$, we are sure that $n^{\prime}(a, 1-0)<+\infty$.

Finally, that $\lim _{a \rightarrow+\infty} n^{\prime}(a, b)=+\infty$ is a consequence of the fact that $\lim _{a \rightarrow+\infty} m_{2}(a, b, n)<0$ for every $b$ and $n$.

It remains to study the mapping $n \mapsto h\left(\Gamma_{n}, \sigma\right)$. To that goal we compute an equation for $h\left(\Gamma_{n}, \sigma\right)$ by considering the subset $\Gamma_{n}^{*} \subset \Gamma_{n}$ which does not contain homogeneous sequences. The dynamical system $\left(\Gamma_{n}^{*}, \sigma\right)$ is a shift of finite type generated by the graph of Figure 9 . The quantity $h\left(\Gamma_{n}^{*}, \sigma\right)$ is the logarithm of

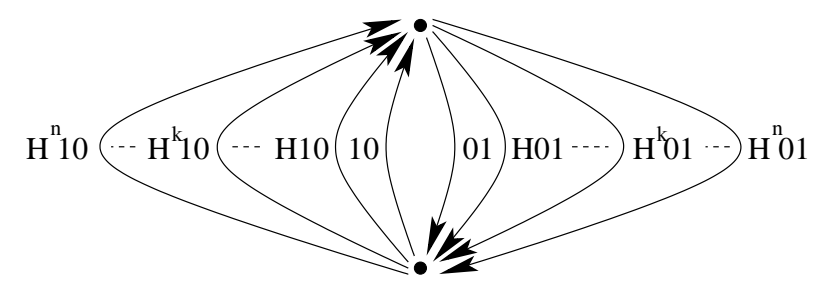

Figure 9: Graph associated with the shift $\left(\Gamma_{n}^{*}, \sigma\right)$ where each $H \in\{00,11\}$ is arbitrary.

the largest root of the equation $\operatorname{det}\left(\operatorname{Id}-M_{n}\left(\frac{1}{z}\right)\right)=0$ where $M_{n}(t)$ is the polynomial matrix [B93] associated with the graph of Figure 9

$$
\left(\begin{array}{cc}
0 & \sum_{k=1}^{n+1} 2^{k-1} t^{k} \\
\sum_{k=1}^{n+1} 2^{k-1} t^{k} & 0
\end{array}\right)
$$

Moreover, the original entropy satisfies $h\left(\Gamma_{n}, \sigma\right)=\max \left\{h\left(\Gamma_{n}^{*}, \sigma\right), \log 2\right\}$.

Using that the largest solution must not be larger than $\frac{1}{2}$, the equation $\operatorname{det}\left(\operatorname{Id}-M_{n}(t)\right)=0$ reduces to $P_{n}(t)=1$ where $P_{n}(t):=\sum_{k=1}^{n+1} 2^{k-1} t^{k} .{ }^{13}$ Finally this polynomial has the following properties

\footnotetext{
${ }^{13}$ In order to compute the entropy of $\Gamma_{n}$, one can also apply the method developed in Appendix B. This method shows that $h\left(\Gamma_{n}, \sigma\right)=-\log x_{n}$ where $x_{n}$ is the unique positive root of $P_{n}-1$.
} 
- $P_{n}\left(\frac{1}{2}\right)>1(n>1)$ and $P_{n}\left(\frac{1}{3}\right)<1$ which imply $\log 2<h\left(\Gamma_{n}, \sigma\right)<\log 3$ when $n>1$. We also have $h\left(\Gamma_{1}, \sigma\right)=\log 2$.

- $P_{n+1}(t)>P_{n}(t)$ which implies $h\left(\Gamma_{n}, \sigma\right)<h\left(\Gamma_{n+1}, \sigma\right)$ and then that the mapping $b \mapsto h\left(\Gamma_{n^{\prime}(a, b)}, \sigma\right)$ is an increasing step function.

- $\lim _{n \rightarrow+\infty} P_{n}\left(\frac{1}{3}\right)=1$ which implies $\lim _{n \rightarrow \infty} h\left(\Gamma_{n}, \sigma\right)=\log 3$ and then $\lim _{a \rightarrow+\infty} h\left(\Gamma_{n^{\prime}(a, b)}, \sigma\right)=\log 3$ for every $b$.

\section{Acknowledgements}

RC thanks the Centre de Physique Théorique, CNRS, for financial support and hospitality. He was partially supported by FCT/POCTI/FEDER, Portugal. BF thanks the Group of Mathematical Physics of the University of Lisbon for financial support and hospitality. PG has been supported by a contract CONICYT PBCT PSD 20.

\section{References}

[ADGW05] F. Antoneli, A.P.S. Dias, M. Golubitsky and Y. Wang, Patterns of synchrony in lattice dynamical systems, Nonlinearity 18 (2005) 2193-2209.

[AF00] V. Afraimovich and B. Fernandez, Topological properties of linearly coupled expanding map lattices, Nonlinearity 13 (2000) 973-993.

[B93] M. Boyle, Symbolic Dynamics and Matrices, in Combinatorial and Graph-Theoretical Problems in Linear Algebra, IMA Volumes in Math. and its Applications 50 (1993) 1-38.

[B01] L. A. Bunimovich, Lattice dynamical systems, in From finite to infinite dimensional dynamical systems, Kluwer Acad. Publ. (2001) 59-83.

[BK06] J-B. Bardet and G. Keller, Phase transition in a piecewise expanding coupled map lattice with linear nearest neighbour coupling, Nonlinearity 19 (2006) 2193-2210.

[C03] S-N. Chow, Lattice dynamical systems in Dynamical Systems, Lect. Notes Math. Springer 1822 (2003) $1-102$.

[CAMTV05] P. Cvitanović, R. Artuso, R. Mainieri, G. Tanner and G. Vattay, Chaos: Classical and Quantum, ChaosBook.org (Niels Bohr Institute, Copenhagen 2005)

[CAV00] R. Carretero-Gonzalez, D. Arrowsmith and F. Vivaldi, One-dimensional dynamics for traveling fronts in coupled map lattices, Phys. Rev. E 61 (2000) 1329-1336.

[CF05] J-R. Chazottes and B. Fernandez ed., Dynamics of coupled map lattices and of related spatially extended systems, Springer Lect. Notes Phys. 671 (2005).

[CF97a] R. Coutinho and B. Fernandez, Extended symbolic dynamics in bistable CML: Existence and stability of fronts, Physica D 108 (1997) 60-80.

[CF97b] R. Coutinho and B. Fernandez, On the global orbits in a bistable CML, Chaos 7 (1997) 301-310.

[CFLM06] R. Coutinho, B. Fernandez, R. Lima and A. Meyroneinc, Discrete time piecewise affine models of genetic regulator networks, J. Math. Bio. 52 (2006) 524-570.

[CKNS04] M. Courbage, V. Kazantsev, V. Nekorkin and M. Senneret, Emergence of chaotic attractor and anti-synchronization for two coupled monostable neurons, Chaos 14 (2004) 1148-1156.

[D99] V. Dobrynskii, Critical sets and properties of endomorphisms built by coupling of two identical quadratic mappings, J. Dynam. Control Systems 5 (1999) 227-254.

[DL05] C.P. Dettmann and D. Lippolis, Periodic orbit theory of two coupled Tchebyscheff maps, Chaos, Solitons and Fractals 23 (2005) 43-54. 
[FG04] B. Fernandez and P. Guiraud, Route to chaotic synchronisation in coupled map lattices: rigorous results, Discrete \& Cont. Dynam. Sys. B 4 (2004) 435-455.

[FJ04] B. Fernandez and M. Jiang, Coupling two unimodal maps with simple kneading sequences, Ergod. Th. Dynam. Sys. 24 (2004) 107-125.

[FM96] L.M. Floria and J.J. Mazo, Dissipative dynamics of the Frenkel-Kontorova model, Adv. Phys. 45 (1996) 505-598.

[GH83] J. Guckenheimer and P. Holmes, Nonlinear oscillators, dynamical systems and bifurcations of vector fields, Springer (1983).

[GM00] G. Gielis and R.S. MacKay, Coupled map lattices with phase transition, Nonlinearity 13 (2000) $867-888$.

[HW79] G.H. Hardy and E.M. Wright, An introduction to the theory of numbers, Fifth edition. Oxford University Press, (1979).

[J02] H. de Jong, Modeling and simulation of genetic regulatory systems: a literature review, J. Compt. Biol. 9 (2002) 69-105.

[JJ02] J. Jost and M. P. Joy, Spectral properties and synchronization in coupled map lattices, Phys. Rev. $\mathrm{E}(3) 65$ (2002) 016201.

[K91] R. Kapral, Discrete models for chemically reacting systems, J. Math. Chem. 6 (1991) 113-163.

[K93] K. Kaneko ed. Theory and applications of coupled map lattices, Wiley (1993).

[KKN92] G. Keller, M. Künzle and T. Nowicki, Some phase transitions in coupled map lattices, Physica D 59 (1992) 39-51.

[KL04] G. Keller and C. Liverani, Coupled map lattices without cluster expansion, Discrete \& Cont. Dynam. Sys. 11 (2004) 325-335.

[KStP01] G. Keller and M. St. Pierre, Topological and measurable dynamics of Lorenz maps, in Ergodic theory, analysis, and efficient simulation of dynamical systems, Springer (2001) 333-361.

[LJ98] S. Lepri and W. Just, Mean field theory of critical coupled map lattices, J. Phys. A: Math. Gen. 31 (1998) 6175-6181.

[LM95] D. Lind and B. Marcus, An introduction to symbolic dynamics and coding, Cambridge University Press (1995).

[MH93] J. Miller and D. A. Huse, Macroscopic equilibrium from microscopic irreversibility in a chaotic coupled-map lattice, Phys. Rev. E 48 (1993) 2528-2535.

[MYDN01] R. Miyazaki, S. Yoshida, Y. Dobashi and T. Nishita, Cloud simulation by CML, Proc. of Pacific Graphics (2001) 363-372.

[PRK01] A. Pikovsky, M. Rosenblum and J. Kurths, Synchronization: A universal concept in nonlinear sciences, Cambridge University Press (2001).

[R99] C. Robinson, Dynamical systems: Stability, symbolic dynamics, and chaos, CRC Press (1999). 